\title{
HEAT TRANSFER IN DESIGN \\ OF ALPHA WASTE STORAGE FACILITIES
}

G. Jansen

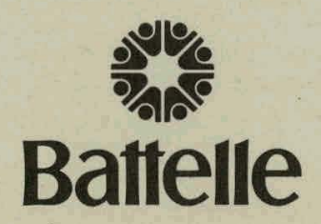

Pacific Northwest Laboratories

Richland, Washington 99352

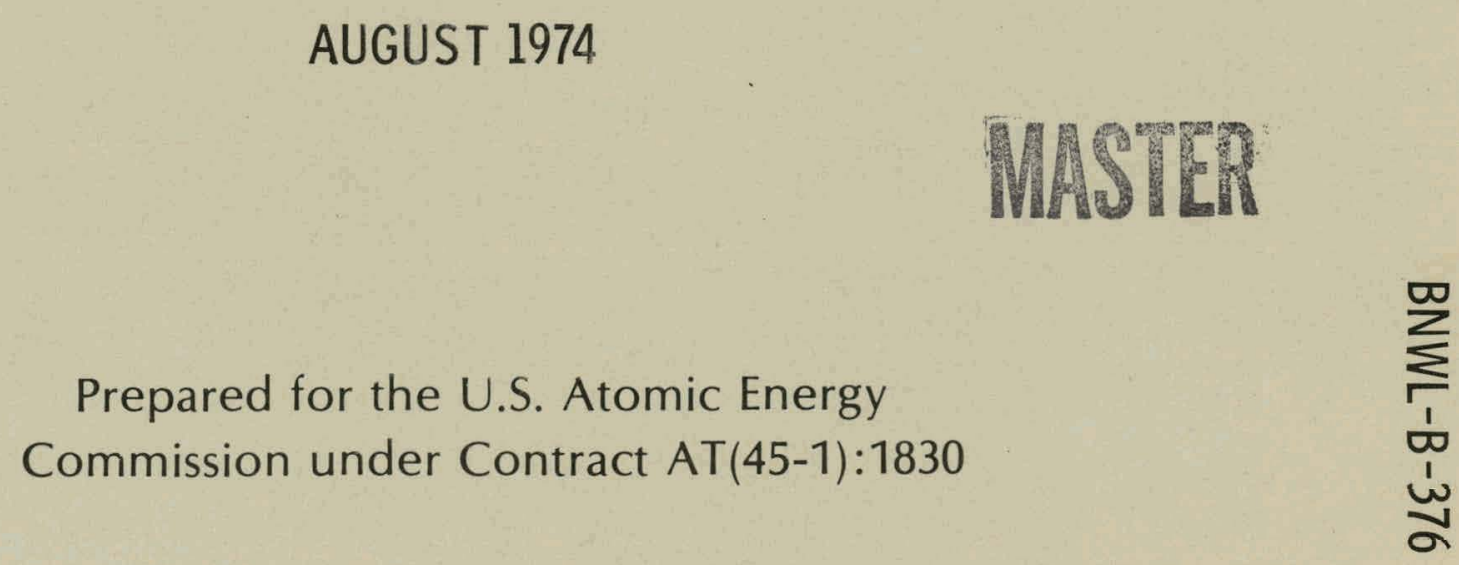




\section{DISCLAIMER}

This report was prepared as an account of work sponsored by an agency of the United States Government. Neither the United States Government nor any agency Thereof, nor any of their employees, makes any warranty, express or implied, or assumes any legal liability or responsibility for the accuracy, completeness, or usefulness of any information, apparatus, product, or process disclosed, or represents that its use would not infringe privately owned rights. Reference herein to any specific commercial product, process, or service by trade name, trademark, manufacturer, or otherwise does not necessarily constitute or imply its endorsement, recommendation, or favoring by the United States Government or any agency thereof. The views and opinions of authors expressed herein do not necessarily state or reflect those of the United States Government or any agency thereof. 


\section{DISCLAIMER}

Portions of this document may be illegible in electronic image products. Images are produced from the best available original document. 


\section{NOTICE}

The report was prepared as an account of work sponsored by the United States Government. Neither the United States nor the United States Atomic Energy Commission, nor any of their employees, nor any of their contractors, subcontractors, or their employees, makes any warranty, express or implied, or assumes any legal liability or responsibility for the accuracy, completeness or usefulness of any information, apparatus, product or process disclosed, or represents that its use would not infringe privately owned rights.

\section{PACIFIC NORTHWEST LABORATORY \\ operated by \\ BATTELLE \\ for the \\ U.S. ATOMIC ENERGY COMMISSION \\ Under Contract AT(45-1)-1830}

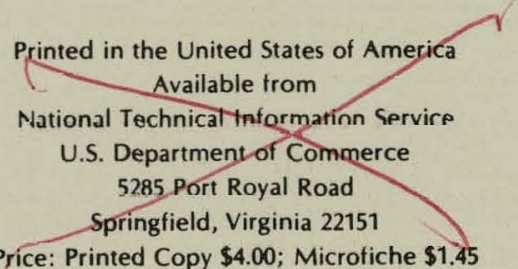




\title{
HEAT TRANSFER IN DESIGN \\ OF ALPHA WASTE STORAGE FACILITIES
}

\author{
by
}

G. Jansen

Nuclear Waste Technology Department

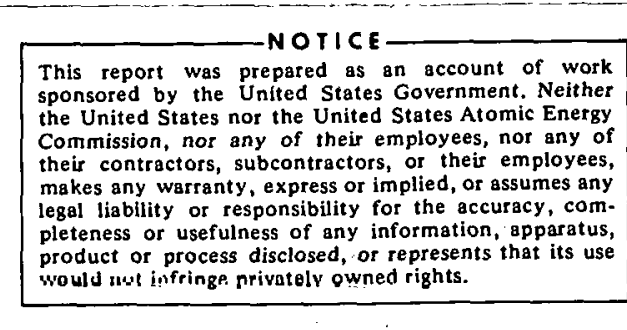

This report is based on work performed under U.S. Atomic Energy Commission Contract No. AT(45-1)-1830.

\section{BATTELLE}

PACIFIC NORTHWEST LABORATORIES

RICHLAND, WASHINGTON 99352 


\section{CONTENTS}

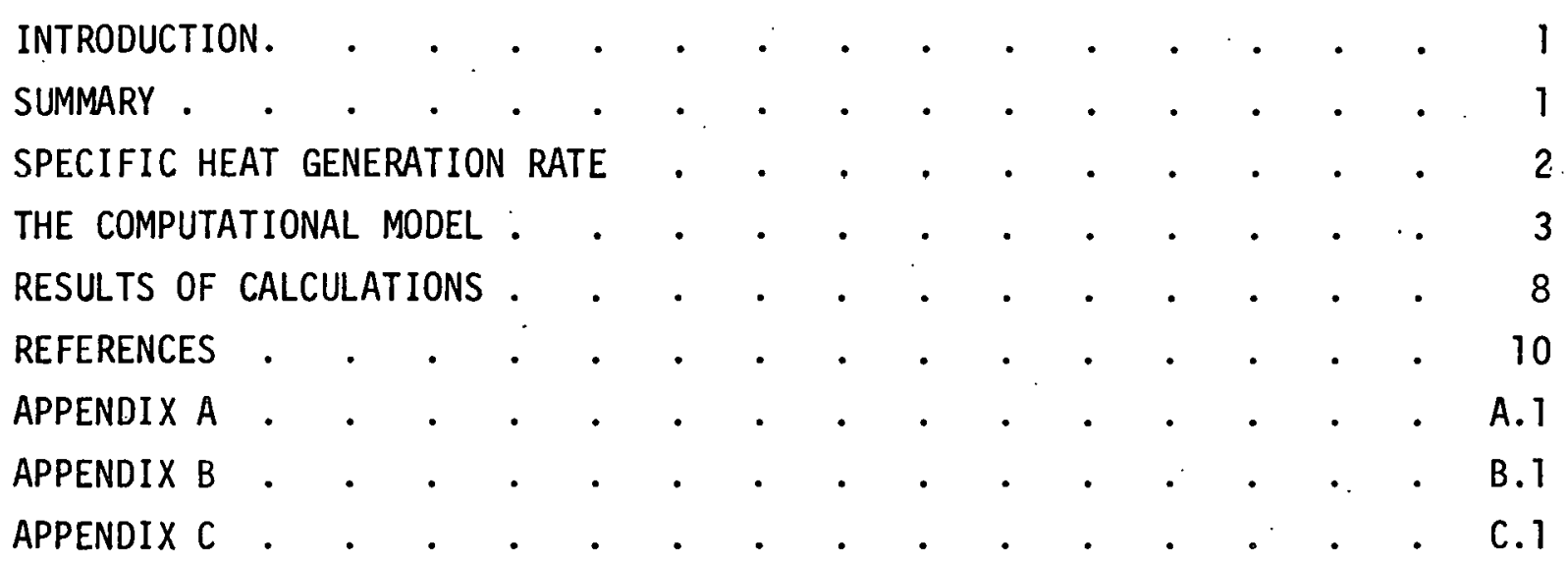




\section{HEAT TRANSFER IN DESIGN \\ OF ALPHA WASTE STORAGE FACILITIES}

G. Jansen

\section{INTRODUCTION}

The work in this report was performed over two years ago at the request of the Atlantic Richfield Hanford Company and was reported to ARHCO by letter on July 26, 1972. (1) Its primary purpose was to provide an estimate of the heat transfer limitations on the spacing and stacking height of storage canisters in an alpha waste storage facility design. This design was one of several concepts then being evaluated by ARHCO for alpha wastes from the U.S. nuclear power fuel cycle.

After 1972, the results of these heat transfer calculations were cited several times within Battelle Northwest for the different purpose of helping to justify a dividing line between high-level and low-level wastes. $(2,3,4,5)$ The dividing value has been used as $0.1 \mathrm{~W} / \mathrm{ft}^{3}$ or $5 \mathrm{~g}$ of plutonium per cubic foot, below which concentration no auxiliary cooling is said to be needed to remain below a temperature limit at which combustible paper and rags will burn in a specified starage design.

Since this dividing value may be reexamined more closely, it seems appropriate to make the modest calculational effort and the detailed bases for the heat transfer model and the storage design configuration available for examination in an internal report.

\section{SUMMARY}

Heat transfer design calculations have been made for a conceptual alpha waste storage facility. Stacks of containers $6 \mathrm{ft}$ by $4 \mathrm{ft}$ by $6 \mathrm{ft}$ high would be placed in a large room with natural dissipation of heat or forced air cooling. The stacks of barrels would be cooled by natural convection flow through vertical channels formed by the 3 -in. radii on the corners of the containers and through 4-in. horizontal gaps between cans. 
The containers would be filled with plutonium-bearing wastes. A BASIC language computer program ALPHAl was developed for estimating temperatures in the alpha waste storage facility. Tentative conclusions arrived at in the study were 1) the maximum heat generation rate in the plutonium was $0.0174 \mathrm{~W} / \mathrm{g}$ at 50 years with a decrease to $0.0087 \mathrm{~W} / \mathrm{g}$ at 500 years because of ${ }^{241} \mathrm{Am}$ growth and decay; 2) stacks up to 10 cans high are permissible; 3) spaces about an inch wide would have to be maintained between the cans to keep the waste cool enough; and 4) even if the walls of the building were 1 -ft-thick concrete, there would be enough heat loss so that forced convection would not be needed.

\section{SPECIFIC HEAT GENERATION RATE}

The initial composition of the plutonium was assumed to be $1 \%{ }^{238} \mathrm{Pu}$, $60 \%{ }^{239} \mathrm{Pu}, 24 \%{ }^{240} \mathrm{Pu}, 11 \%{ }^{241} \mathrm{Pu}, 4 \%{ }^{242} \mathrm{Pu}$ with minor amounts of ${ }^{232} \mathrm{U},{ }^{236} \mathrm{Pu}$, ${ }^{241} \mathrm{Am},{ }^{242} \mathrm{Cm}$, and ${ }^{244} \mathrm{Cm}$ present. The initial heat generation rate is 0.01 $\mathrm{W} / \mathrm{g} \mathrm{Pu}$, principally due to the ${ }^{239} \mathrm{Pu}$ and ${ }^{240} \mathrm{Pu}$. As the plutonium ages, the heat generation rate increases as shown in Table 1 because of ${ }^{241} \mathrm{Pu}$ decay to ${ }^{241} \mathrm{Am}$. A maximum heat generation rate of $0.0175 \mathrm{~W} / \mathrm{g} \mathrm{Pu}$ is reached at 50 years, after which a gradual decrease occurs as the ${ }^{241}$ Am decays. Tables A.1 and A.2 of Appendix A give a detailed breakdown of the plutonium composition versus time from 1 to 1000 years after reactor discharge. Minor isotope concentrations are given for illustrative purposes only and are not calculated from any real situation.

TABLE 1. Heat Generation Rate of Alpha Wastes

\begin{tabular}{ccccc} 
Time, years & W/gPu & & Time, years & W/g Pu \\
\cline { 2 - 2 } 1 & 0.0100 & & 60 & 0.0174 \\
2 & 0.0103 & & 70 & 0.0172 \\
5 & 0.0115 & 80 & 0.0170 \\
10 & 0.0132 & 90 & 0.0167 \\
20 & 0.0155 & 100 & 0.0163 \\
30 & 0.0167 & 200 & 0.0134 \\
40 & 0.0173 & 500 & 0.0087 \\
50 & 0.0175 & 1000 & 0.0052
\end{tabular}


THE COMPUTATIONAL MODEL

A BASIC language computer program named ALPHAI, listed in Appendix C, was written in order to make. the computations. Input data are listed and explained with the program.

Initially, the specific heat generation rate of the plutonium is calculated as a function of the initial composition and the age of the waste. The volumetric heat generation rate is then estimated and the total heat generation rate in the facility is estimated from the number of cans present. A steady-state energy balance on the facility determines the required air rate for a given temperature rise in a room containing well mixed air:

$$
\Delta T_{R O O M}=\frac{q \cdot M_{P u}-\frac{K_{C} A_{R}}{X_{2} \Delta T_{R O O M}}}{\rho_{1} F_{1} c_{1}}
$$

where

$$
\begin{aligned}
M_{P u}^{q} & =\text { heat generation rate per gram } P u \\
K_{C} & =\text { thermal conductivity of concrete wall } \\
A_{R} & =\text { surface area of room } \\
X_{2} & =\text { concrete wall thickness } \\
\rho_{1} C_{1} & =\text { volumetric heat capacity of air } \\
F_{1} & =\text { flow rate of air. }
\end{aligned}
$$

The waste cans have a $6 \mathrm{ft}$ by $4 \mathrm{ft}$ cross section and are $6 \mathrm{ft}$ tall. (See Figures 1 and 2 supplied hy ARHCO for the can arrangement.) They are stacked with I-beams between layers of cans, so that there is a horizontal space between each level, and they may be spaced so closely together that the only vertical channel for cooling is the space formed by the 3-in. radius of curvature at the corners of the cans. Alternatively, the cans may be spaced apart enough to allow additional convective cooling all along the vertical walls. 


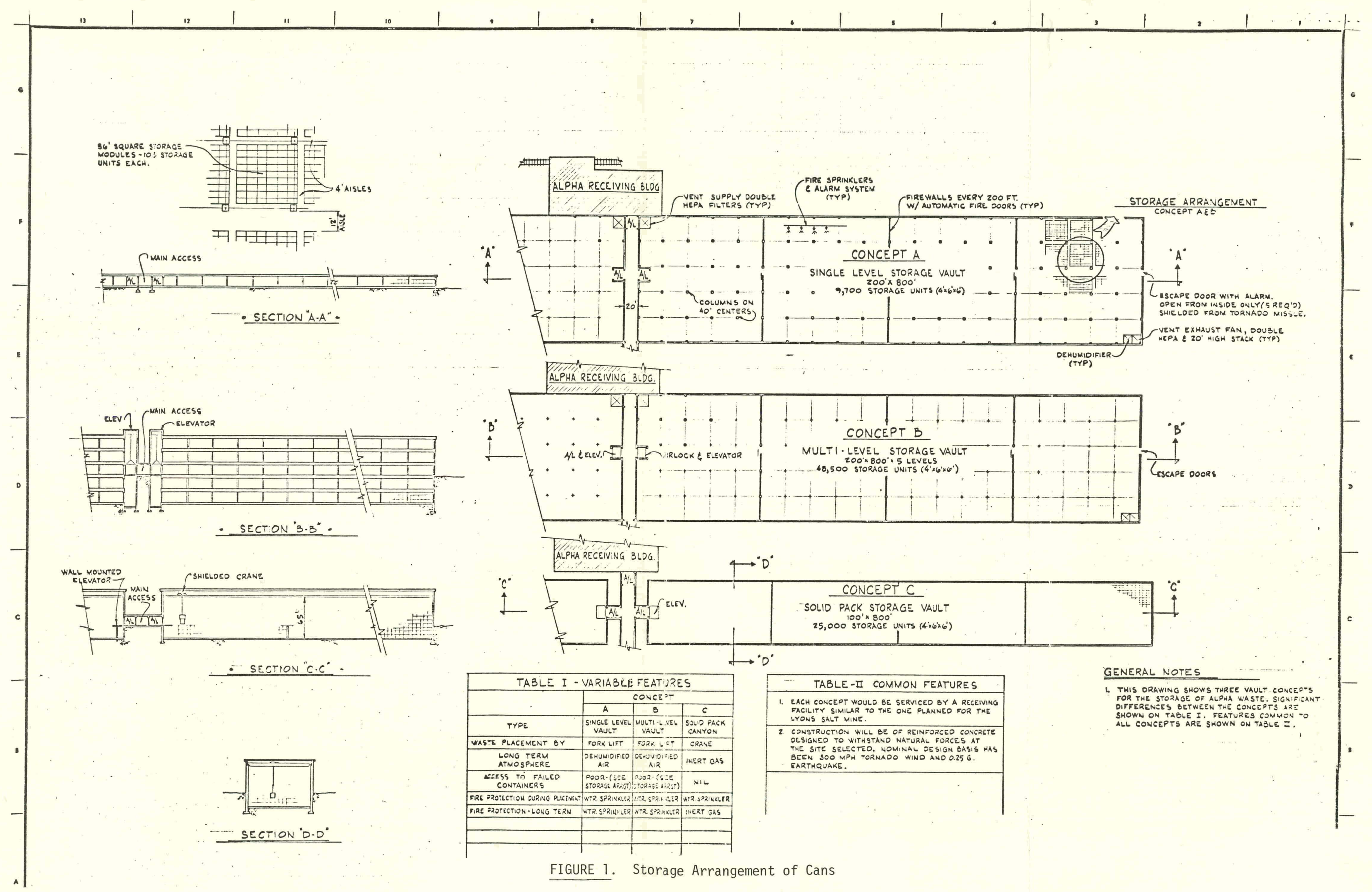




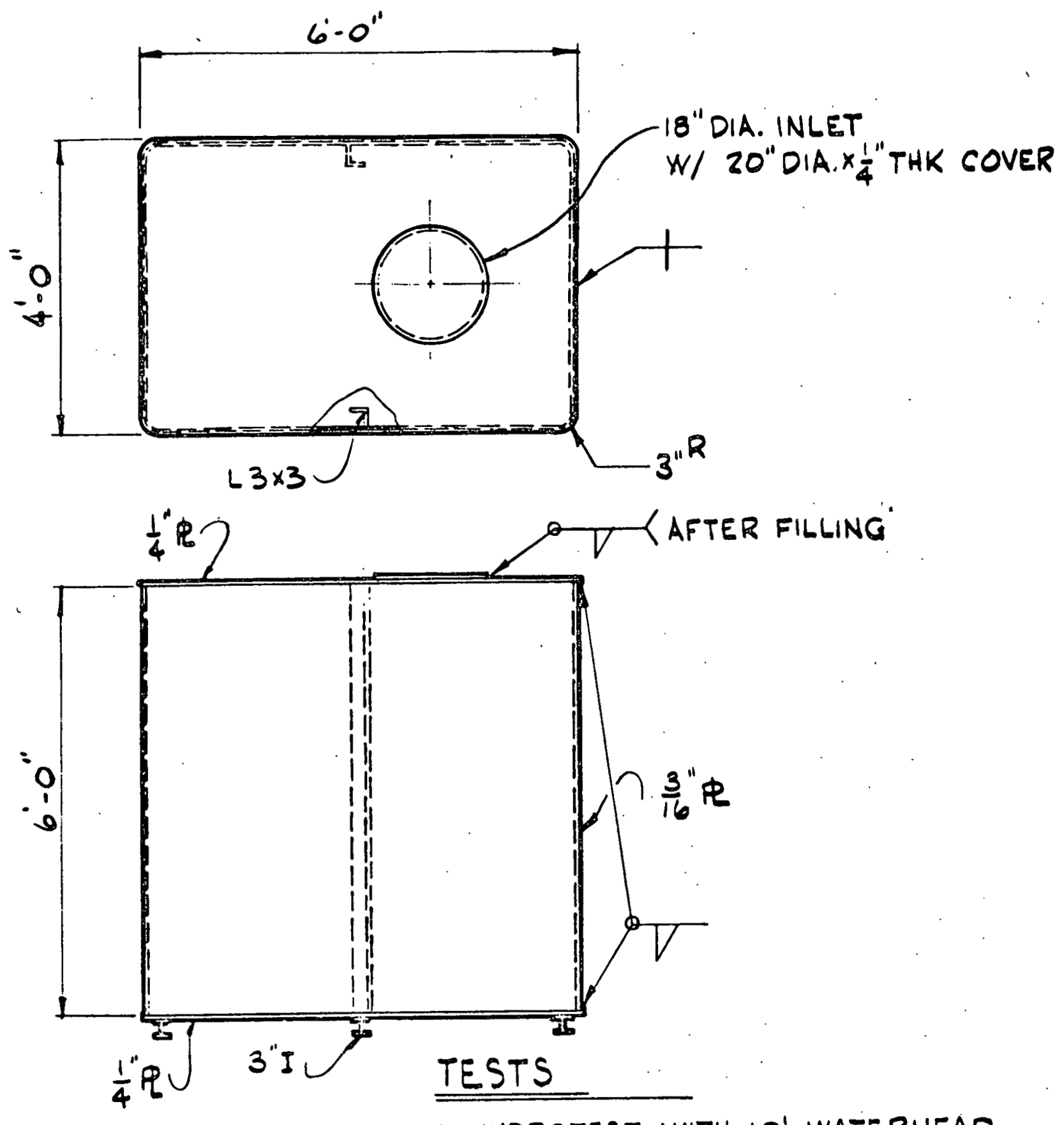

1. HYDROTEST WITH 10' WATERHEAD ABOVE TOP $R$. NO VISIBLE LEAKS IN 2 HOURS.

2. 4'DRUP TEST HALF FULL OF WATER. NO VISIBLE LEAKS IN 2 HOURS.

FIGURE 2. Standard Container Design (Alpha Engineered Storage Facility)

5 
It was assumed that air would be allowed to circulate by natural convection vertically in the channels between the cans, with the hydraulic equivalent diameter of the channel being calculated from the expression:

$$
D_{e}=2 r_{e}=\frac{\text { cross-sectional area of channel }}{\text { perimeter of channel }}
$$

and with an equivalent length of $3 \mathrm{ft}$ of channel being added due to expansion of the flow stream in each space between layers of cans. An expression for the average natural convection heat transfer coefficient from the can wall to the air is then given by Kreith: ${ }^{(6)}$

$$
\bar{h}_{c}=\frac{K_{s}}{r_{e}} * 0.42\left(\frac{r_{e}}{L_{e}} * G r * \operatorname{Pr}\right) 1 / 4
$$

where $\bar{h}_{c}$ is the average heat transfer coefficient from the wall of the can to the air

$K_{S}$ is the thermal conductivity of air

$L_{e}$ is the equivalent length of the stack of cans

$\mathrm{Gr}$ and $\mathrm{Pr}$ are the Grashof and Prandt7 numbers, respectively.

The mass flow rate of the air through the channel was estimated from Equations 9 to 10a, page 219, of McAdams. (7)

$$
G=\left[\frac{\bar{h}_{c} D_{e}}{K_{s}} *(P r)^{-0.4}\left(\frac{\mu}{D_{e}}\right)^{0.8} * \frac{1}{0.023}\right]^{1.25} \frac{1 b}{h_{r ~ f t}{ }^{2}}
$$

This equation was developed for forced convection but should also be suitable for relating the mass flow rate to the heat transfer coefficient in closed channels regardless of the driving force.

The temperature rise of the air as it passes vertically up the channel is

$$
\Delta T_{\text {CHANNEL }}=\frac{S_{0} A_{C} L}{G^{*} A{ }^{C}{ }^{C}}
$$


where $S_{0}$ is the volumetric heat generation rate in the waste

$A_{C}$ is the vertical surface area of the can

$A_{c h}$ is the cross-sectional area of the channel.

This temperature rise was arbitrarily assumed to be additive to the room temperature rise to give the maximum air temperature at the top of the uppermost can in the stack. The temperature rise from the air to the can wall is

$$
\Delta T_{W A L L}=\frac{M_{1}}{A_{6} \bar{h}_{c} L_{0}}
$$

where $\quad M_{1}$ is the heat generation rate in one can

$A_{6}$ is the can perimeter

$L_{0}$ is the can length.

Heat flows simultaneously to the air in the channel by three parallel paths: If the cans have no space between them, it flows directly to the channel through the waste, through the waste to the ends of the can into the air space above and below the can and then into the channel, and through the waste to the vertical can wall and horizontally within the thick metal wall to the channel. These parallel paths are represented by

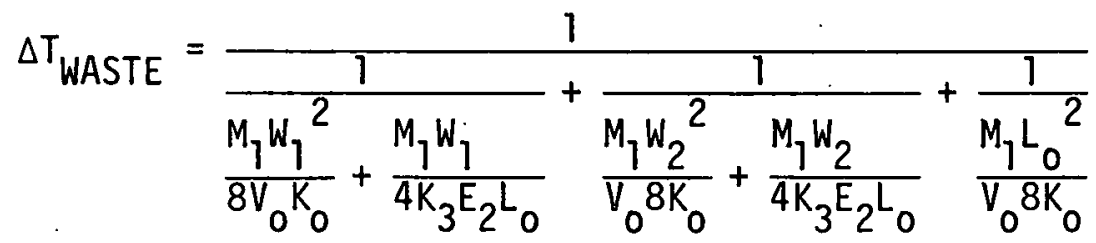

where $\quad W_{1}$ is the 4-ft width of can

$W_{2}$ is the 6-ft width of can

$L_{0}$ is the 6-ft length of one can

$V_{0}$ is the can volume

$K_{u}$ is the waste thermal conductivity

$K_{3}$ is the metal wall thermal conductivity

$E_{3}$ is the can wall thickness. 
If spaces are present between the cans, the heat flows directly into the channel, and

$$
\Delta T_{\text {WASTE }}=\frac{M_{1}}{V_{0} K_{0}} \frac{1}{\frac{8}{W_{1}^{2}}+\frac{8}{W_{2}^{2}}+\frac{8}{L_{0}^{2}}}
$$

The total temperature rise is then approximated by

$$
\Delta T_{\text {TOTAL }}=\Delta T_{\text {ROOM }}+\Delta T_{\text {CHANNEL }}+\Delta T_{\text {WALL }}+\Delta T_{\text {WASTE }}
$$

\section{RESULTS OF CALCULATIONS}

The results are presented in Tables B.1, B.2 and B.3 of Appendix B. In Table B.1, the required air rates and the temperature rises are presented as functions of the number of cans in a stack (2,5 and 10) for a completely insulated room and with no space between the can walls. The waste thermal conductivity of $0.3 \mathrm{Btu} /(\mathrm{hr})\left(\mathrm{ft}^{2}\right)\left({ }^{\circ} \mathrm{F} / \mathrm{ft}\right)$ is typical of paper or ashes with dead air space, and the plutonium loading of $5 \mathrm{~g} / \mathrm{ft}^{3}$ is a maximum value.

A typical result for $50-y r$ cooling gives a required air rate of $1377 \mathrm{scfm}$ for a room temperature rise of $20^{\circ} \mathrm{F}$. For a 10-can-high stack the natural convection air flow rate per channel is $3.5 \mathrm{scfm}$, and the total temperature rise to the center of the waste of $185^{\circ} \mathrm{F}$ is comprised of $146^{\circ} \mathrm{F}$ in the rising air and $19.2^{\circ} \mathrm{F}$ within the waste itself. The rise at the gas film at the wall is less than $1^{\circ} \mathrm{F}$.

In Table B.2 the results of increasing the space between cans from 0 to $0.10 \mathrm{ft}$ are shown. For the case above, the total temperature rise is $47^{\circ} \mathrm{F}$ with $16.4^{\circ} \mathrm{F}$ due to the rising air column and $10.5^{\circ} \mathrm{F}$ in the waste itself. Clearly, a small space between cans such as might be obtained by using corrugations is beneficial. 
In Table B.3 the temperature rise is estimated with heat losses from the facility through $26,000 \mathrm{ft}^{2}$ of 1 -ft-thick concrete walls. Forced air cooling is not required to maintain room temperature rises below $20^{\circ} \mathrm{F}$ in all cases.

The foregoing analysis was made assuming extreme condtions. A plutonium waste facility would not be expected to contain $5.0 \mathrm{~g} / \mathrm{Pu} / \mathrm{ft}^{3}$ in every can because of criticality considerations. The results do show that heat generation is not a problem if a little space between cans is allowed. Program ALPHAI can readily be used for a wide range of desired design conditions upon demand. 


\section{REFERENCES}

1. G. Jansen, Battelle-Northwest to D. E. Larson, ARHCO, personal communication, July 26, 1972.

2. Staff of Battelle-Northwest, Program for Management of Hazardous Wastes for Environmental Protection Agency Office of Solid Waste Management Programs, PB233-630, PB233-631, Contract No. 68-01-0762, JuTy 1973.

3. W. K. Winegardner and J. D. Kaser, Radioactive Waste Management for Nuclear Energy Center - Prel iminary, BNWL-B-334, January 1974.

4. G. Jansen, K. J. Schneider, and V. L. Hammond, A Conceptual System for Handling Alpha-Bearing Wastes, BNWL-SA-5001, presented at the Seminar on the Management of Plutonium-Contaminated Solid Wastes, OECD Nuclear Energy Agency, Marcoule, France, October 1974.

5. L. D. Williams, Battelle-Northwest, to G. Jansen, Battelle-Northwest, Private Communication, September 13, 1974.

6. F. Kreith, Principles of Heat Transfer, p. 317, International Textbook Company, 1958.

7. W. H. McAdams, Heat Transmission, Third Edition, McGraw-Hill Book Company, 1954 . 
APPENDIX A

TABLE A.1. PLUTONIUM COMPOSITIONS versus COOLING TIME Range: 1,10 , $50,100,200,500,1000 \mathrm{yr}$

TABLE A.2. PLUTONIUM COMPOSITIONS versus COOLING TIME Range: $1,2,5$, $10,20,30,40,50,60,70$, $80,90 \mathrm{yr}$ 


\section{TABLE A.1. PLUTONIUM COMPOSITIONS versus COOLING TIME}

\begin{tabular}{|c|c|c|c|}
\hline AL & $\begin{array}{l}\text { nS/27/72 } \\
\text { WASTF STIPAGF FAC } \\
\text { AT TRANG FFP ANMIYYS }\end{array}$ & TY & \\
\hline 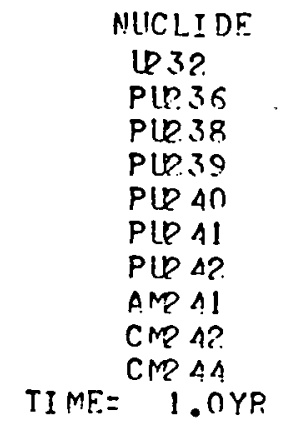 & 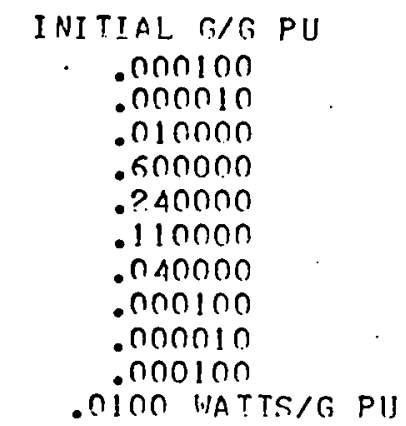 & 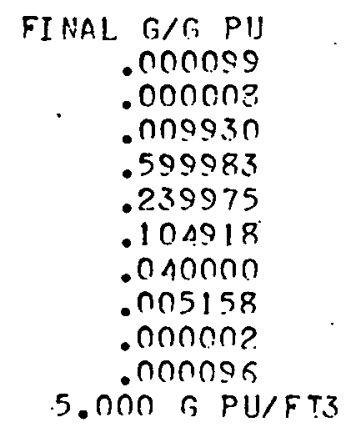 & $\begin{array}{l}1, A T T S / G P I 1 \\
6.807 E-05 \\
1.450 E-04 \\
5.551 E-03 \\
1.140 E-03 \\
1.6 \Delta A E-03 \\
4.407 E-04 \\
4.60 \cap E-06 \\
5.927 E-04 \\
2.587 E-04 \\
2.734 F-04 \\
388 K G P U\end{array}$ \\
\hline 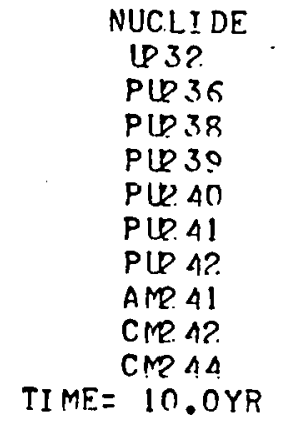 & 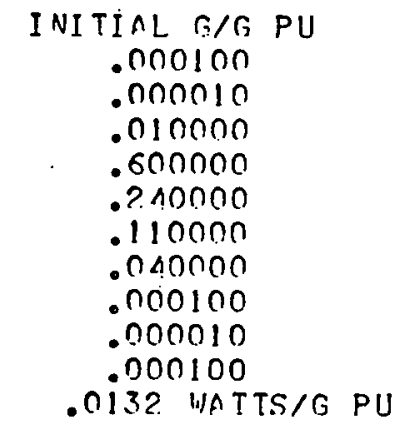 & $\begin{aligned} & \text { FINAL } \text { S/G PU } \\
& . 000091 \\
& .000001 \\
& .009260 \\
& .599830 \\
& .239754 \\
& .068512 \\
& .039999 \\
& .041040 \\
& .000000 \\
& .000068 \\
& 5.000 \text { G PU/FT3 }\end{aligned}$ & $\begin{array}{l}\text { WATTS/G PU } \\
6.243 E-05 \\
1.62 .5 E-05 \\
5.176 E-03 \\
1.140 E-03 \\
1.642 E-03 \\
2.879 E-04 \\
4.600 E-06 \\
4.716 E-03 \\
2.230 E-10 \\
1.939 E-04 \\
388 K G \mathrm{KU}\end{array}$ \\
\hline $\begin{array}{l}\text { NUCLIDE } \\
\text { Q.32. } \\
P Q 36 \\
P Q 38 \\
P Q 39 \\
P Q 40 \\
P Q 41 \\
P Q 42 \\
A M 41 \\
C P 242 .\end{array}$ & $\begin{array}{l}\text { INITIAL rIf PU } \\
.000100 \\
.000010 \\
.010000 \\
.600000 \\
. ? 40000 \\
.110000 \\
.040000 \\
.000100 \\
.000010\end{array}$ & $\begin{aligned} & \text { FINAL } \text { G/G PU } \\
& .000062 \\
& .000000 \\
& .006781 \\
& .599149 \\
& .238773 \\
& .010332 \\
& .039996 \\
& .094130\end{aligned}$ & $\begin{array}{l}\text { WATTS/G PIJ } \\
4.2 .49 E-05 \\
9.711 E-10 \\
3.791 E-03 \\
1.138 E-03 \\
1.636 E-03 \\
4.340 E-05 \\
4.600 E-06 \\
1.082 E-02\end{array}$ \\
\hline $\begin{array}{c}\text { CMR 44 } \\
\text { IIME }=50.0 Y R\end{array}$ & $\begin{array}{l}\text { nOn100 } \\
.0175 \text { WATTS/G. PU }\end{array}$ & $\begin{array}{l}.000015 \\
5.000 \text { G PU/FT3 }\end{array}$ & $\begin{array}{l}\text { 4.?. I } \triangle F-\cap 5 \\
388 \mathrm{KGPU}\end{array}$ \\
\hline 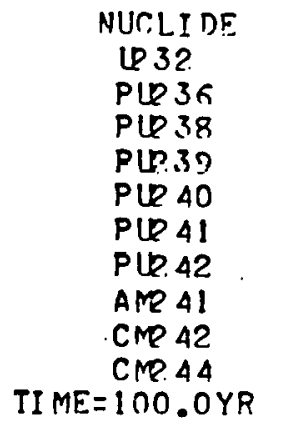 & $\begin{array}{l}\text { INITIAL F/G PU } \\
.000100 \\
.000010 \\
.010000 \\
.600000 \\
.240000 \\
.110000 \\
.040000 \\
.000100 \\
.000010 \\
\text { OONO100 } \\
.0163 \text { WATTS/G PU }\end{array}$ & $\begin{array}{c}\text { FINAL G/G PU } \\
.000038 \\
.000000 \\
.004594 \\
.598299 \\
.237553 \\
.000971 \\
.039993 \\
.095731 \\
.000000 \\
.000002 \\
\text {.0.0ก r PU/FT3 }\end{array}$ & $\begin{array}{l}\text { WATTS/G PU } \\
2.62 .7 E-05 \\
5.099 E-15 \\
2.568 E-03 \\
1.137 E-03 \\
1.627 E-03 \\
4.076 E-06 \\
4.599 E-06 \\
1.100 E-02 \\
.000 E+00 \\
6.253 E-06 \\
388 \text { KG PIJ }\end{array}$ \\
\hline
\end{tabular}

A. 1 


\title{
TABLE A.1. (Continued)
}

\author{
ALPHAI $\quad 16: 48 \quad 05 / 27 / 72$ \\ ALPHA UASTF. STUPAGE FACILITY \\ HEAT TRANSFER ANALYSIS
}

\begin{tabular}{|c|c|c|c|c|}
\hline 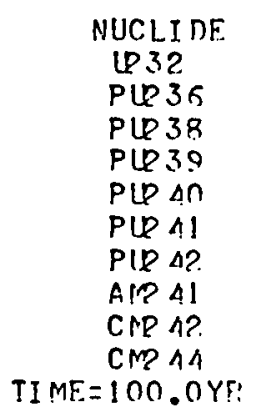 & 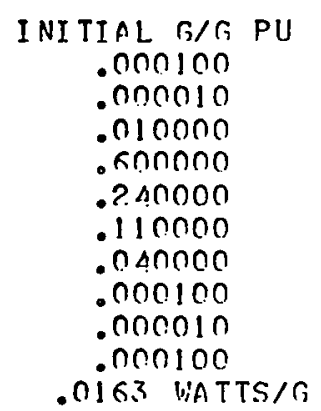 & $P U$ & 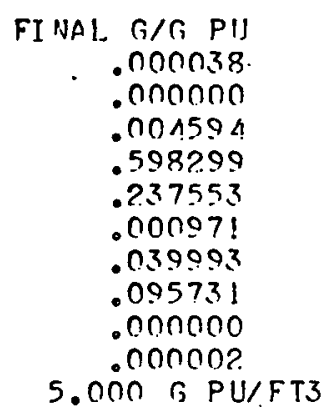 & $\begin{array}{l}\text { UATTS/G PU } \\
2.627 E-05 \\
5.099 E-15 \\
2.068 E-0.3 \\
1.137 E-0.3 \\
1.627 E-03 \\
4.076 E-06 \\
4.599 E-06 \\
1.100 E-02 \\
.000 E+00 \\
6.253 E-06 \\
388 \mathrm{KG} \mathrm{PU}\end{array}$ \\
\hline 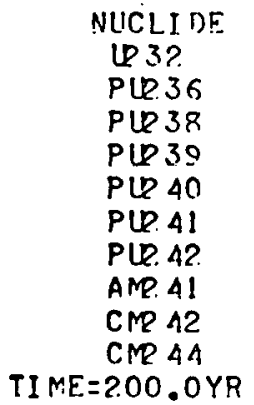 & $\begin{array}{l}\text { INITIAL G/G PU } \\
.000100 \\
.000010 \\
.010000 \\
.600000 \\
.240000 \\
.110000 \\
.040000 \\
.000100 \\
.000010 \\
.000100 \\
.013 A \text { WATTS/G }\end{array}$ & PU & $\begin{array}{c}\text { FI NAL G/F PIJ } \\
.000015 \\
.000000 \\
.002108 \\
.596604 \\
.235130 \\
.000009 \\
.039985 \\
.082429 \\
.000000 \\
.000000 \\
5.000 \text { G PU/FT3 }\end{array}$ & $\begin{array}{l}\text { WATTS/G PU } \\
1.00 \triangle E-05 \\
1.406 E-25 \\
1.178 E-03 \\
1.134 E-03 \\
1.611 E-03 \\
3.596 E-08 \\
4.598 E-06 \\
9.471 E-03 \\
.000 E+00 \\
1.377 E-07 \\
388 K G \text { KU }\end{array}$ \\
\hline $\begin{array}{l}\text { NUCLIDE } \\
\text { U.32. } \\
\text { PU } 36 \\
\text { PU } 38 \\
\text { PL } 39 \\
\text { PLQ } 40 \\
\text { PID } 41 \\
\text { PUQ 42. } \\
\text { AMP } 41 \\
\text { CMQ 42. } \\
\text { CMP } 44 \\
\text { IME= } 500.0 Y R\end{array}$ & $\begin{array}{c}\text { INITIAL G/G PU } \\
.000100 \\
.000010 \\
.010000 \\
.600000 \\
.240000 \\
.110000 \\
.010000 \\
.000100 \\
\text {.0NO010 } \\
\text { ONOOIOO } \\
\text { ON87 UATTS/A }\end{array}$ & & $\begin{array}{c}\text { FINAL } G / G P U \\
.000001 \\
.000000 \\
.000204 \\
.591545 \\
.228011 \\
.000000 \\
.039963 \\
.051029 \\
.000000 \\
.000000 \\
\text {.000 } 5 \text { PU/FTS }\end{array}$ & $\begin{array}{l}\text { WATTS/G PU } \\
5.604 E-07 \\
.000 E+00 \\
1.139 E-01 \\
1.12 .4 F-03 \\
1.562 . E-03 \\
2.470 E-14 \\
4.5065-06 \\
5.863 E-03 \\
.000 E+00 \\
1.470 .-12 \\
388 \mathrm{kli})\end{array}$ \\
\hline 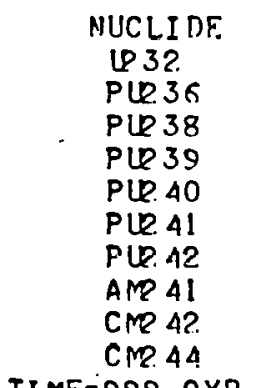 & $\begin{array}{l}\text { INITIAL G/G PU } \\
\text {. } 00100 \\
.000010 \\
.010000 \\
.600000 \\
.240000 \\
.110000 \\
.040000 \\
.000100 \\
.000010 \\
.000100\end{array}$ & & $\begin{array}{r}\text { FINAL } F / G \text { PU } \\
.000000 \\
.000000 \\
.000004 \\
.5832 .26 \\
.216643 \\
.000000 \\
.039927 \\
.022978 \\
.000000 \\
.000000\end{array}$ & $\begin{array}{l}\text { WATTS/G PU } \\
4.614 E-09 \\
.000 E+00 \\
2.335 E-06 \\
1.108 E-03 \\
1.454 E-03 \\
1.385 E-24 \\
4.592 E-06 \\
2.640 E-03 \\
.000 E+00 \\
7.004 E-21\end{array}$ \\
\hline
\end{tabular}


TABLE A.2. PLUTONIUM COMPOSITIONS versus COOLING TIME

\begin{tabular}{|c|c|c|c|c|c|}
\hline & & $\begin{array}{l}\text { WASTE STURAGE } \\
T \text { TRANSFER ANAL }\end{array}$ & & LITY & \\
\hline . & 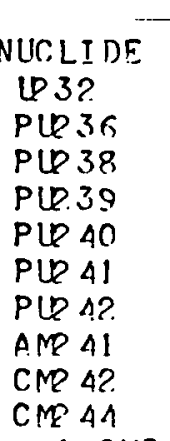 & $\begin{array}{l}\text { INITIAL G/G PU } \\
.000100 \\
.000010 \\
.010000 \\
.600000 \\
.240000 \\
.110000 \\
.040000 \\
.000100 \\
.000010 \\
.0 n 0100\end{array}$ & $\cdot$ & $\begin{aligned} \text { FINAL } & \text { r/G PU } \\
. & 000059 \\
. & 000008 \\
. & 009930 \\
. & 599983 \\
. & ? 39975 \\
. & 104918 \\
. & 010000 \\
. & 005158 \\
. & 000002 \\
. & 000096\end{aligned}$ & $\begin{array}{l}\text { WATTS/G PU } \\
6.807 E-05 \\
1.450 E-04 \\
5.551 E-03 \\
1.140 E-03 \\
1.644 E-03 \\
4.407 E-04 \\
4.600 E-06 \\
5.92 .7 E-04 \\
2.587 E-04 \\
2.734 E-04\end{array}$ \\
\hline TIME= & $=1.0 Y F$ & .0IOO WATTS/G & $P U$ & $5.000 \mathrm{G} \mathrm{PU} / F \mathrm{T3}$ & 388 KG P \\
\hline . & 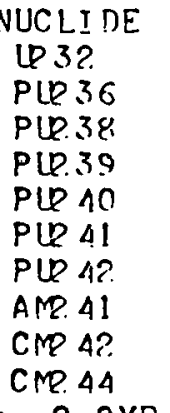 & 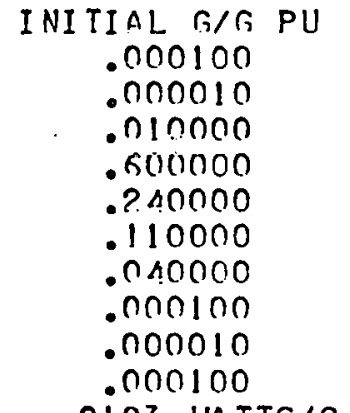 & . & 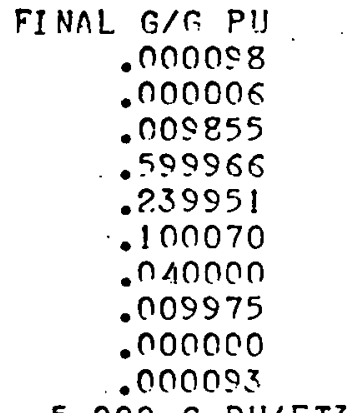 & $\begin{array}{l}\text { WATTS/G PU } \\
6.742 . E-05 \\
1.137 E-04 \\
5.509 E-03 \\
1.140 E-03 \\
1.644 E-03 \\
4.203 E-04 \\
4.600 E-06 \\
1.146 E-03 \\
5.483 E-05 \\
2.631 E-04\end{array}$ \\
\hline & 2. $\quad$ OYP & .0103 UIATTS/G & PU & 5.000 G PU/FT3. & 388 K.G P \\
\hline N & 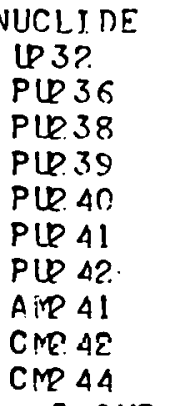 & $\begin{array}{c}\text { INITIAL G/G PU } \\
.000100 \\
.000010 \\
.010000 \\
.600000 \\
.240000 \\
.110000 \\
.040000 \\
.000100 \\
.000010 \\
.000100\end{array}$ & & $\begin{aligned} & \text { FINAL } \text { F/F PU } \\
& .000095 \\
& .000003 \\
& .009628 \\
& .599915 \\
& .239877 \\
& .08623 ! \\
& .040000 \\
& .023082 \\
& .000000 \\
& .000083\end{aligned}$ & $\begin{array}{l}\text { WATTS/G PU } \\
6.550 E-05 \\
5.483 E-05 \\
5.382 E-03 \\
1.140 E-03 \\
1.643 E-03 \\
3.647 E-04 \\
4.60 \cap E-06 \\
2.652 E-03 \\
5.2 .18 E-07 \\
2.347 E-04\end{array}$ \\
\hline TIMF:= & : $\quad 5.0 Y R$ & .0115 WATTS/G & & $5.000 G \mathrm{PU} / \bar{F} \mathrm{T3}$ & . $388 \mathrm{KG} \mathrm{P}$ \\
\hline & 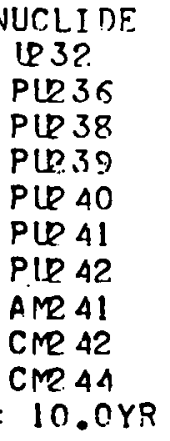 & $\begin{array}{c}\text { INITIAL } F / G \text { PIJ } \\
.000100 \\
.000010 \\
.010000 \\
.600000 \\
.2 .40000 \\
.110000 \\
.040000 \\
.000100 \\
.000010 \\
.000100 \\
.0132 \text { WATTS/G }\end{array}$ & & $\begin{aligned} & \text { FINAL } \text { G/G PI! } \\
& .000091 \\
& .000001 \\
& .009260 \\
& .590830 \\
& .239754 \\
& .068512 \\
& .039999 \\
& .041040 \\
& .000000 \\
& .000068 \\
& 5.000 \text { G PU/FT3 }\end{aligned}$ & $\begin{array}{l}\text { 'UATS/G PU } \\
6.243 E-05 \\
1.62 .6 E-05 \\
5.176 E-03 \\
1.140 E-03 \\
1.642 E-03 \\
2.879 E-04 \\
4.60 \cap E-06 \\
4.716 E-03 \\
2.230 E-10 \\
1.939 E-04 \\
388 \mathrm{KG} P\end{array}$ \\
\hline
\end{tabular}

A. 3 
TABLE A.2. (Continued)

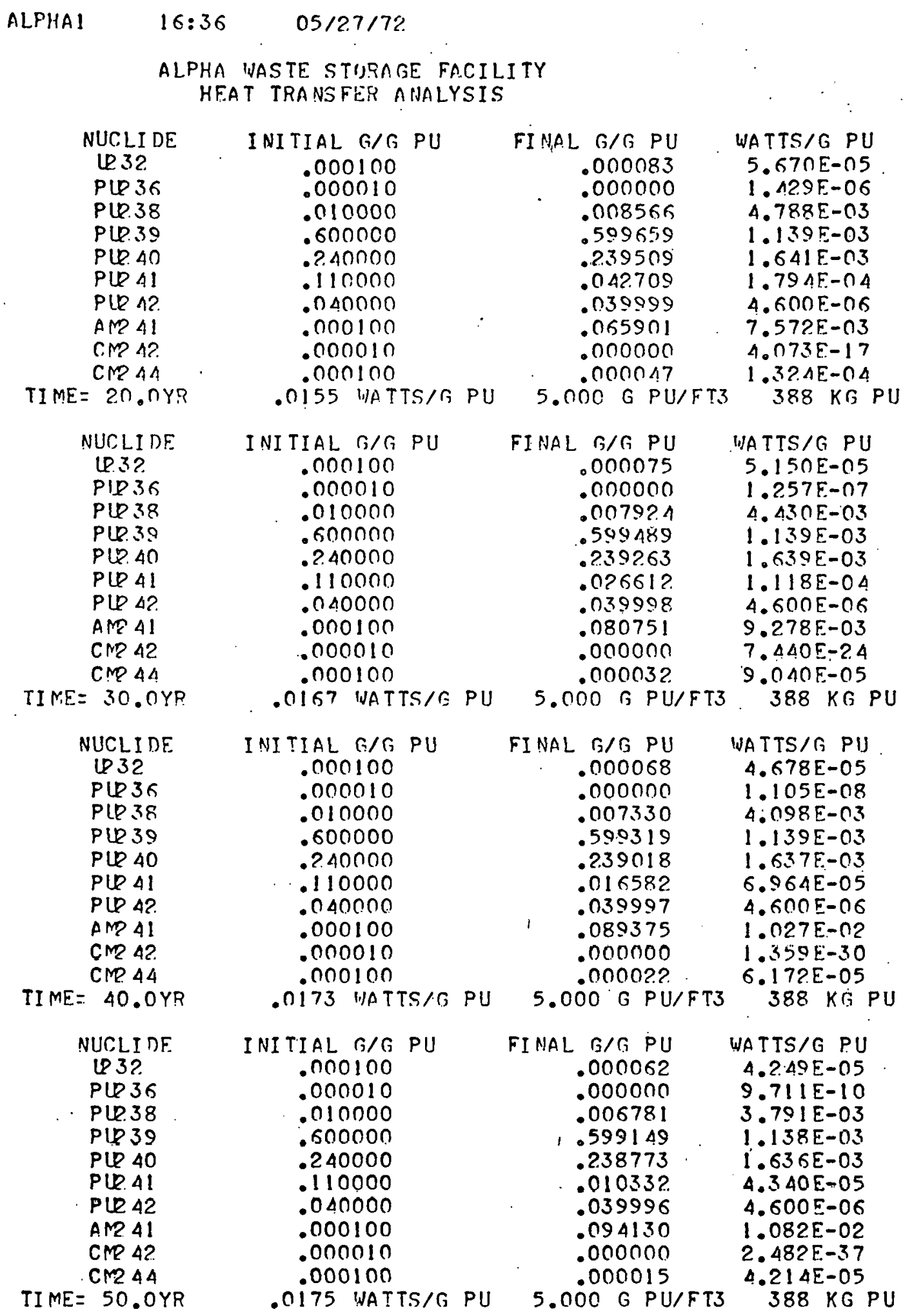

A. 4 
TABLE A.2. (Continued)

ALPHA $1 \quad 16: 12.05 / 27 / 72$

ALPHA WASTE STISRAE FACILITY

HEAT TRANSFER ANALYSIS

NUCLIDE

U32.

$P L .36$

$P L 238$

PLP39

$P L P 40$

$P L P A 1$

PLQ 42 .

AMP 41

CMP 42

CMP 41

TIME: $=60.0 Y ?$

NUCLITDE

L23?.

PLP 36

PL 38

PLR39

PLP 40

PLL 41

PLP 12

$A M \triangle 1$

CMR 42 .

CMP $\triangle \triangle$

TIMF: $=70.0 \mathrm{YR}$

NUCLI DE

lP 32

PL 36

$P 1838$

PID 39

PL 40

PLV 41

PLE 42.

AMR 41

CMe 42 .

CMQ 44

TI ME $=80.0 Y R$

NUCLIDF

प3?

PLI6

$P L 38$

PLP 39

PUL 40

PLQ 41

PLP 42

AMP 41

CMR 42.

CMP 44

TIME $=90.0 Y R$
I NI TIAL G/G PU

.000100

. 000010

- 0100no

- sonona

- 2.40000

.110000

- 040000

- nnolon

.000010

.000100 .

.0171 IATTS/G PU

INITIAL $F / G \quad P U$

- 000100

. 000ก10

.010000

- 600000

. 2.40000

.110000

.040000

. 00100

.000010

.000100

.0172 WATTS/G PU

INITIAL $B / G P U$

- חOO100

.000010

.010000

. 600000

.240000

.110000

.040000

.000100

- noonia

- nnoló

.0170 INATTS/G PU

INITIAL G/G PU

- 000100

.000010

- n! 0000

: 600000

-2. 4 inno0

.110000

.040000

.000100

.000010

.000100

.0167 WATTS/F PU
FINAL G/G PU

.000056

.000000

.0062 .73

.598979

.2 .3852 .9

.006438

.039996

. 096482

.000000

.000010

$5.000 \mathrm{G} \mathrm{PU/FT3}$

UATTS/G PU

$3.859 E-05$

8. $537 \mathrm{E}-11$

$3.507 \mathrm{E}-03$

$1.138 \mathrm{E}-\mathrm{C3}$

1. $634 \mathrm{~F}-03$

$2.704 E-05$

4. 599E-06

1.109E-02.

$.000 E+00$

2. $877 \mathrm{E}-0.5$

388 KG PU

FINAL G/G PU

.000051

.000000

.005803

.598809

.238284

.004012

.039995

.097348

.000000

WATTS/G PU

$3.505 E-05$

$7.505 E-12$.

$3.244 E-03$

$1.138 E-03$

1. $632 E-03$

1. $685 E-05$

4. 599E-06

1. $119 E-02$

. $000 E+00$

$.00000 ?$

1. $965 E-05$

5. กOO G PU/FT3 $388 \mathrm{KG}$ PU

FINAL G/G PU .000046

INATTS/G PU

. 000000

.005368

.598639

.238040

. 002500

.039904

.097297

- nnonona - 0nonos

5. OON G PIJ/FT3

$3.184 E-05$

6. 598E-13

3. $001 E-03$

$1.137 E-03$

1. $631 E-03$

1. $050 E-05$

4. 599E-06

1.118F- -02 .

ONOE+OO

$1.341 E-05$

$388 \mathrm{KG} \mathrm{PU}$

FINAL G/F PU

.000042.

.00000 ?

WATTS/G PU

.004966

.598469

.237796

.001558

.039903

.096684

.000000

.000003

5.000 G PU/FT3

$2.892 E-05$

5. $800 E-14$

2. $776 E-0.3$

$1.137 \mathrm{E}-03$

1. $62.9 E-03$

$6.542 E-06$

$4.509 E-06$

1.111E-02.

- NOOE+0O

9.1595-06

388 KG PU 
APPENDIX B

RESULTS OF ALPHA WASTE STORAGE HEAT TRANSFER ANALYSIS

TABLE B.1. TEMPERATURES IN INSULATED ROOM, WITH AIR FLOW. NO SPACE BETWEEN CANS.

Range of Variables:

Stack Height: 2, 5, 10 cans

Cooling Time: $1,10,50,100 \mathrm{yr}$

Room Temperature Rise: $5,10,20^{\circ} \mathrm{F}$

TABLE B.2. TEMPERATURES IN INSULATED FACILITY WITH

AIR FLOW. 0.1 INCH SPACE BETWEEN CANS.

Range of Variables: Same as in Table B.I

TABLE B.3. TEMPERATURES IN A FACILITY WITH HEAT. LOSSES THROUGH A 1-FT THICK CONCRETE WALL.

Range of Variables:

Can Spacing: $0,0.1$ in.

Stack Height: 10 cans

Cooling Time: $1,10,50,100 \mathrm{yr}$ 
TABLE B.1. TEMPERATURES IN INSULATED ROOM, WITH AIR. FLOW. NO SPACE BETWEEN CANS.

\author{
AI.PIIA1 $\quad 16: 10 \quad 05 / 2.7 / 7 ?$ \\ ALPHIA UASTE STURAGE FACILITY \\ HEAT TRANSFER ANALYSIS
}

TUTAL NUMRER UF BARRELS= $5 A M$ IN CUNFIGURATIUN 9 BY $3 \cap$ BY 2 HIGH BARRELS APE 6 FT BY 4 FT BY 6 FT LUNG WITH .2.5 FT RADIUS AT CURNERS SPACING IS .OO FT HURIZINNTAL AND 0.33 FT VERTICAL

SUPFACF AREA IF RUIM $=15000$ FT? WITH I FT THICK WALLS WITH K=.00 VULIIIAE UF BA?REL $=143.7$ FT3, TUTAL WASTE VULUME $=77586$ FT3

THFPMAL CUNDUCTI UITY UF WASTE .03 RTU/HR/FT2/(DEG F/FT)

CAN WALL IS .18.7 IN. THICK WITH THFRMAL CUNDUCTIVITY $=14.0$

\section{TI}

\begin{tabular}{|c|c|c|c|c|c|c|}
\hline $\begin{array}{l}\text { RUIM } \\
\text { RISF } \\
\text { DES F F } \\
5.00 \\
10.00 \\
20.00\end{array}$ & $\begin{array}{c}\text { AIR } \\
\text { RATE } \\
\text { SCFM } \\
3168.1 \\
1584.1 \\
792.0\end{array}$ & $\begin{array}{l}\text { WALL } \\
\text { RISF } \\
\text { DET F } \\
.180 \\
1180 \\
.180\end{array}$ & $\begin{array}{l}\text { STACK } \\
\text { RISE } \\
\text { DES F } \\
37.128 \\
37.096 \\
37.065\end{array}$ & $\begin{array}{l}\text { WASTE } \\
\text { RISE } \\
\text { DEG F } \\
11.0 A 6 \\
11.0 A 6 \\
11.0 \triangle 6\end{array}$ & $\begin{array}{l}\text { MAX. } \\
\text { WASTF } \\
\text { DEG F } \\
53.173 \\
58.14 ? \\
68.110\end{array}$ & $\begin{array}{l}C(\text { SNV. } \\
F L(1,) \\
\text { SCFM } \\
1.58 \\
1.58 \\
1.58\end{array}$ \\
\hline
\end{tabular}

IIME $=10.0 Y R$

$\begin{array}{ll}\text { RIJJM } & \text { AIR } \\ \text { RISE } & \text { RATE } \\ \text { DEF F } & \text { SCFM } \\ 5.00 & 4153.7 \\ 10.00 & 2.076 .9 \\ 20.00 & 1038.4\end{array}$

TIME $=50 . \cap Y R$

$\begin{array}{lc}\text { RUIM } & \text { AIR } \\ \text { RISE } & \text { RATE } \\ \text { DEG F } & \text { SCFM } \\ 5.00 & 5507.3 \\ 10.00 & 2.753 .6 \\ 20.00 & 1376.8\end{array}$

TI ME $=100.0 Y R$

$\begin{array}{ll}\text { RUSM } & \text { AIR } \\ \text { RISE } & \text { RATE } \\ \text { DES F } & \text { SC.FM } \\ 5.00 & 5153.0 \\ 10.00 & 2576.5 \\ 20.00 & 12.88 .2\end{array}$
NoIN AT END

.0132. hatTs/g PU

$\begin{array}{ll}\text { WALL } & \text { STACK } \\ \text { RISF } & \text { PISE } \\ \text { DEGT } & \text { DEG F } \\ .2 .2 .4 & 45.503 \\ .2 .2 .4 & 45.465 \\ .2 .23 & 45.426\end{array}$

.0175 WATTS/G PU

$\begin{array}{ll}\text { WALL } & \text { STACK } \\ \text { RISE } & \text { RISE } \\ \text { DEFF } & \text { DEF F } \\ .280 & 56.239 \\ .2 .80 & 56.191 \\ .280 & 56.143\end{array}$

.0163 WATTS/G PIJ

$\begin{array}{ll}\text { WALL } & \text { STACK } \\ \text { RISE } & \text { RISE } \\ \text { DEFF } & \text { DEG F } \\ .266 & 53.499 \\ .2 .66 & 53.454 \\ .265 & 53.409\end{array}$

$16: 14$

\begin{abstract}
RAN $O$ MINS
\end{abstract}
0.28 SECS 


\section{TABLE B.1. (Continued)}

ILPHAI $\quad 16: 15 \quad 05 / 27 / 72$

\section{ALPHA WASTE STURAGE FACILITY \\ HEAT TRANSFER ANALYSIS}

TUTAL NUMBER IJF RARRELS= 540 IN CONFIGUPATIUN 9 BY 12. BY 5 HIGH BARRELS ARF 6 FT BY 4 FT BY 6 FT LUNG UITH .2.5 FT RADIUS AT CIJPNERS SPACING IS .INO FT HURIZIJNTAL AND 0.33 FT VERTICAL

SURFACE AREA UF RUJUM= 15000 FT2 WITH 1 FT THICK WALLS WITH $K=.00$

VIJLUIAE UF BARREL $=143 . ?$ FT3, TUTAL WASTF VISLUME $=77586$ FT3

THERPAL CUNDIJCTIVITY UF WASTE .03 BTU/HR/FT2/(DEG F/FT)

CAN WALL IS .187 IN. THICK WITH THERMAL CONNUCTIUITY $=14.0$

TIME $=1.0 Y F_{1}$

. IInO hatTS/F PU 5.000 g PU/FT3 38E KG PU

$\begin{array}{lllllll}\text { RIJUM } & \text { HIR } & \text { WALL } & \text { STACK } & \text { WASTE } & \text { MAX. } & \text { CIJNY. } \\ \text { RISE } & \text { RATF } & \text { RISF } & \text { RISE } & \text { RISF } & \text { WASTF } & \text { FLIJW } \\ \text { DEG } & \text { SCFM } & \text { DEGF } & \text { DEGF } & \text { DFGF } & \text { DF.FF } & \text { SCFM } \\ 5.00 & 3168.1 & .127 & 67.2 .15 & 11.0 A 6 & 83.2 .60 & 2.18 \\ 10.00 & 1584.1 & .125 & 65.663 & 11.046 & 86.709 & 2.23 \\ 20.00 & 792.0 & .125 & 65.608 & 11.046 & 96.653 & 2.24\end{array}$

TIME $=10.0 Y R$

\section{RIJUM}

RISE

DEG $F$

5.00

10.00

20.00

\section{AIR}

RATE

SCFM

1153.7

2076.9

1038.4

TIME: $=50.0 Y R$

$\begin{array}{ll}\text { RUIJM } & \text { AIR } \\ \text { RISE } & \text { RATE } \\ \text { DEF F } & \text { SCFM } \\ 5.00 & 5507.3 \\ 10.00 & 2753.6 \\ 20.00 & 1376.8\end{array}$

TIME $=100.0 Y R$

$\begin{array}{ll}\text { RIJUM } & \text { AIR } \\ \text { RISE } & \text { RATE } \\ \text { DEGF } & \text { SCFM } \\ 5.00 & 5153.0 \\ 10.00 & 2575.5 \\ 2.0 .00 & 1288 . ?\end{array}$
NIJW AT END

16:19 RAN O M IS

\section{.0132 . haTTS/G PU}

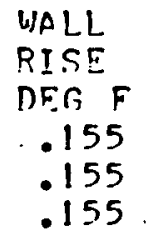
STACK
RIST.
DEG F
80.544
80.476
80.108

.0175 WATTS/G PU

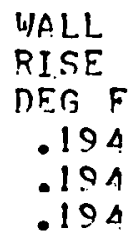

STACK

RISF

DEG $F$

99.547

99.463

99.379

.0163 watTS/G PU

!ALL

RISE

DEF F

.184

.184

.184 $\begin{array}{ll}\text { STACK } & \text { WASTE } \\ \text { RISE } & \text { RISE } \\ \text { DEF F } & \text { DEG F } \\ 94.697 & 17.966 \\ 94.617 & 17.966 \\ 94.537 & 17.966\end{array}$
5.000

G PUIFT3

$388 \mathrm{KG} P U$

WASTE
RISE
DEG F
14.482
14.482
14.482

MAX.
WASTE
DES F
100.026
104.958
114.890

C()NV.

FL(נ)

SCFM

2.39

2.39

5.000 G PU/FT3 $388 \mathrm{KG} \mathrm{PU}$

WASTE
RISE
DEF F
19.201
19.201
19.201
maX. WASTE
$D E G F$
123.748
128.664
13.8 .580

CONV.

FLoly

SCFM

2. .56

2.56

2.57

5.000 G PULFT3 .388 KG PU

$\begin{array}{ccl}\text { WASTE } & \text { MAX. } & \text { CIJNV. } \\ \text { RISE } & \text { WASTF } & \text { FLI)! } \\ \text { DEGF } & \text { DFG F } & \text { SCFM } \\ 17.966 & 117.663 & 2.52 \\ 17.966 & 122.583 & 2.52 \\ 17.966 & 132.503 & 2.52\end{array}$

0.28 SECS 


\section{TABLE B.1. (Continued)}

ALPHA1 $\quad 15: 35 \quad 05 / 2.7 / 72$

\section{ALPIIA UIASTF. STURAGE TACILITY \\ HEAT TRANSFEP ANALYSIS}

TUTAL NIJMBER IF RARRELS $=540$ IN CUNFIFIJPATIUN : BY. 6 BY IO HIGH BARRELS ARE 6 FT RY $\triangle$ FT BY 6 FT LUNS WITH .2.5 FT RADIUS AT CISRNERS SPACING IS .OO FT HURIZIJNTAL AND 0.33 FT-VEOTICAL

SURFACF. AREA UF ROSOM= 15000 FT? WITH I FT THICK WALLS WITH $K=$. OO

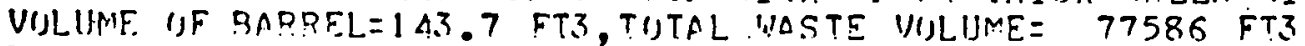

THERMAL CUNDUCTIVITY IJF WASTE. OB RTU/HR/FT2./(DFG F/FT)

CAN WALL IS .187 IN. THICK WI TH THERMAL CUNDUCTIVITY $=14.0$

\begin{tabular}{|c|c|c|c|c|c|c|c|}
\hline IIMF: $=$ & & .01004 & IS/G PU & $5.0 n \cap \mathrm{G}$ & PU/FT3 & 388 & $\mathrm{KGPU}$ \\
\hline $\begin{array}{l}\text { RIJUM } \\
\text { RISE } \\
\text { DEG F F } \\
5.00 \\
10.00 \\
20.00\end{array}$ & $\begin{array}{c}\text { AIR } \\
\text { RATF } \\
\text { SCFM } \\
3168.1 \\
158.4 .1 \\
792.0\end{array}$ & $\begin{array}{l}\text { WALL } \\
\text { RIS5 } \\
\text { DEG F } \\
.096 \\
.007 \\
.097\end{array}$ & $\begin{array}{l}\text { STACK } \\
\text { RISE } \\
\text { DEG F } \\
98.584 \\
98.918 \\
99.254\end{array}$ & $\begin{array}{l}\text { WASTE } \\
\text { RISF } \\
\text { DEF } \\
11.0 \Delta 6 \\
11.0 \Delta 6 \\
11 \\
1.0 \Delta 6\end{array}$ & 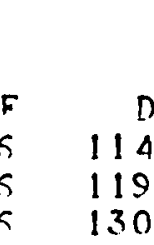 & $\begin{array}{l}X . \\
S T E \\
F \\
30 \\
64 \\
99\end{array}$ & $\begin{array}{l}\text { CINV } \\
\text { FL1W } \\
\text { SCFM } \\
2.98 \\
2.97 \\
2.96\end{array}$ \\
\hline I $M E=1$ & YR & 0132,14 & S/G PUI & $5.000 \mathrm{G}$ & $P U / F T 3$ & 38,8 & KG PU \\
\hline $\begin{array}{l}\text { RIJUM } \\
\text { RISF } \\
\text { DES F } \\
5.00 \\
10.00 \\
20.00\end{array}$ & $\begin{array}{c}\text { AIR } \\
\text { RATE } \\
\text { SCFP } \\
4153.7 \\
2076.0 \\
1038.4\end{array}$ & $\begin{array}{l}\text { MALL } \\
\text { RISE } \\
\text { DEST F } \\
.120 \\
.1 ? 0 \\
.117\end{array}$ & $\begin{array}{l}\text { STACK } \\
\text { DISE } \\
\text { DES } \\
120.66 A \\
121.073 \\
117.735\end{array}$ & $\begin{array}{l}\text { UASTE } \\
\text { PISE } \\
\text { DFS } \\
14 . \Delta Q ? \\
14.48 ? \\
14 . \Delta 82\end{array}$ & $\begin{array}{r}0 \\
140 \\
145 \\
152\end{array}$ & $\begin{array}{l}X . \\
\text { STE } \\
\text { ST } \\
\Delta 6 \\
55 \\
17\end{array}$ & $\begin{array}{l}\text { CUNN. } \\
F L U 11 \\
\text { CCFM } \\
3.19 \\
3.18 \\
3.27\end{array}$ \\
\hline$I M E=5$ & & $.0175 \mathrm{w}$ & $S / G P U$ & $5.000 \mathrm{r}$ & PIJ/FT3 & 388 & $K G P U$ \\
\hline $\begin{array}{l}\text { RIJUM } \\
\text { RISE } \\
\text { DES F } \\
5.00 \\
10.00 \\
20.00\end{array}$ & $\begin{array}{c}\text { AIR } \\
\text { RATE } \\
\text { SCFM } \\
5507.3 \\
2.753 .6 \\
1.376 .8\end{array}$ & $\begin{array}{l}\text { WALL. } \\
\text { RISE } \\
\text { DEF F } \\
.150 \\
.147 \\
147\end{array}$ & $\begin{array}{l}\text { STACK } \\
\text { RISE } \\
\text { NES F } \\
148.926 \\
145.636 \\
145.513\end{array}$ & $\begin{array}{l}\text { WASTE } \\
\text { PISF } \\
\text { DES } \\
19.201 \\
19.201 \\
19.201\end{array}$ & $\begin{array}{l}.0 \\
173 \\
174 \\
184\end{array}$ & $\begin{array}{l}X . \\
\text { STE } \\
F \\
27 \\
37 \\
14\end{array}$ & $\begin{array}{l}\text { CIJNV. } \\
\text { FLIJ11 } \\
\text { SCFM } \\
3.42 \\
3.50 \\
3.50\end{array}$ \\
\hline $1 M E=1 O C$ & & $.0163 \quad$ W & {$[S / G$ PUI } & $5.0 \cap 0 \mathrm{r}$ & PII/FT3 & 388 & $K G P U$ \\
\hline $\begin{array}{l}\text { RUIJM } \\
\text { RISF. } \\
\text { DE.G F } \\
5.0 n \\
10.00 \\
2.0 .00\end{array}$ & $\begin{array}{c}\text { AIR } \\
\text { RATE } \\
\text { SCFM } \\
5153.0 \\
2576.5 \\
1288 . ?\end{array}$ & $\begin{array}{l}\text { WALL } \\
\text { RISE } \\
\text { DEFF F } \\
.142 \\
.139 \\
.139\end{array}$ & $\begin{array}{c}\text { STACK } \\
\text { RISE } \\
\text { DEF F } \\
111.717 \\
138.541 \\
138.42 .4\end{array}$ & $\begin{array}{l}\text { WASTE } \\
\text { RISE } \\
\text { DEF F } \\
17.966 \\
17.066 \\
17.966\end{array}$ & $\begin{array}{r}7 \\
164 \\
166 \\
176\end{array}$ & $\begin{array}{l}X . \\
S T F \\
F \\
8.3 \\
07 \\
90\end{array}$ & $\begin{array}{l}\text { COUNY. } \\
\text { FLOUG) } \\
\text { SC.FM } \\
3.37 \\
3.49 \\
3.45\end{array}$ \\
\hline
\end{tabular}

$15: 39$ RAN O MINS 0.48 SECS 


\title{
TABLE B.2. TEMPERATURES IN INSULATED FACILITY WITH AIR FLOW.
}

\subsection{IN. SPACE BETWEEN CANS}

\author{
ALPHAI $16: 05 \quad 05 / 2.7 / 7 ?$ \\ ALPHA WASTF STURAGE FACILITY \\ HEAT TRAISFER ANALYSIS
}

TUTAL NUMBER UF BARRELSE 540 IN CIJNFI GIIRATIUN 9 RY 30 BY 2. HI GH BARRELIS ARE 6 FT BY 4 FT BY 6 FT LUNIG UITH 2.5 FT RADIUS AT CURNERS SPACING IS .10 FT HUPIT.UITAL AND 0.33 FT VERTICAL

SURFACE AREA UF PIJU: $=15000$ FT2 WITH I FT THICK WALIS UITH K=.00 VIJLUME UF RARREL=143.7 FT3, TITRL WASTIE VIJLUME= 7758.6 FT3

THERMAL COUNDICTIVITY IJF UASTF .OZ RTU/HR/FT2/(DER F/FT)

CAN WALI. IS .!97 IN. THICK WI TH THERIALL CINDUSTIVITY $=14.0$

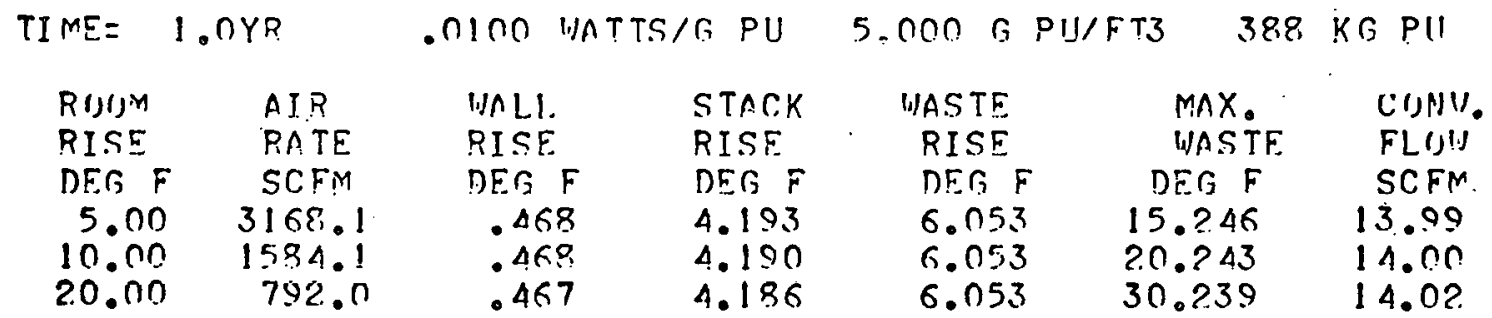

II ME $=10.0 Y R$

.0132 . UATTS/G PU

5.000 G PU/FT3 $388 \quad K G$ PU

$\begin{array}{lllllll}\text { RIJIM } & \text { AIR } & \text { WALI. } & \text { STACK } & \text { WASTE } & \text { MAX. } & \text { C(JNY. } \\ \text { RISE } & \text { RATE } & \text { RISE } & \text { RISF } & \text { RISE } & \text { WASTE } & \text { FLIJW } \\ \text { DEG F } & \text { SCFM } & \text { DEFF } & \text { DEGF } & \text { DEG F } & \text { DEGF } & \text { SCFW } \\ 5.00 & 415.3 .7 & .581 & 5.139 & 7.936 & 18.075 & 14.97 \\ 10.00 & 2.076 .9 & .581 & 5.135 & 7.936 & 23.071 & 14.98 \\ 20.00 & 1038.4 & .583 & 5.158 & 7.936 & 33.094 & 14.91\end{array}$

TIME: $=50.0 Y R$

$\begin{array}{ll}\text { RIJUM } & \text { AIR } \\ \text { RISE } & \text { RATF } \\ \text { DEF F } & \text { SCFM } \\ 5.00 & 5507.3 \\ 10.00 & 2753.6 \\ 20.00 & 1376.8\end{array}$

.0175 WATTS/G PIJ

5.000 G PUFT3 $388 \mathrm{KG}$ PU

$\begin{array}{lllcr}\text { WALL } & \text { STACK } & \text { WASTE } & \text { MAX. } & \text { CIJNV. } \\ \text { RISE } & \text { RISE } & \text { RISE } & \text { WASTE } & \text { FLIJW } \\ \text { DEG F } & \text { DEGF } & \text { DEFF } & \text { DEGF } & \text { SCFM } \\ .72 .9 & 6.351 & 10.52 .2 & 2.1 .874 & 16.06 \\ .728 & 6.346 & 10.52 .2 & 26.858 & 16.017 \\ .731 & 6.373 & 10.522 & 36.895 & 16.00\end{array}$

TIME: $=100.0 Y R$

.0163 VIATTS/G PIJ

5.000

G PU/FT3

$388 K G P U$

$\begin{array}{lllllll}\text { RIJM } & \text { AIR } & \text { WALL } & \text { STACK } & \text { WASTE } & \text { MAX. } & \text { CIJNV. } \\ \text { RISE } & \text { RATE } & \text { RISE } & \text { RISF } & \text { RISE } & \text { WASTE } & \text { FLIIU } \\ \text { DEGF } & \text { SCFM } & \text { DEGF } & \text { DEFF } & \text { DEG F } & \text { DEGF } & \text { SCFM } \\ 5.00 & 5153.0 & .691 & 6.042 & 9.845 & 20.887 & 15.79 \\ 10.00 & 2576.5 & .691 & 6.037 & 9.845 & 25.882 & 15.81 \\ 20.00 & 1288.2 & .693 & 6.063 & 9.845 & 35.908 & 15.74\end{array}$
NIJU A.T FND

16:09 RAN O MINS 0.2. SECS 


\title{
TABLE B.2. (Continued)
}

ALPHA1 $\quad 16: 1905 / 27 / 72$

\author{
ALPHA WASTE STURAGE FACILITY \\ HEAT TRANSFER ANIALYSIS
}

IUTAL NUMPER IJF RAPRELS= 540 IN CIUNFI GIJPATIUN 9 RY I2. RY 5 HIGH BARRELS ARE 6 FT P.Y 4 FT RY 6 FT LINNF WITH .25 FT PADIUS AT CURNERS SPACING IS .10 FT HURIZIJ!ITAL AND 0.33 FT VERTICAL

SURFACF. AREA IJF RUYM= 15000 FT? UITH I FT THICK WALLS WITH K= .OO VIJLUIME IJF BARPF.L $=143.7$ FT3, TIJTAL WASTE. VISLUMF= 77586 FT3 THFRMAL CUNDUCTIVITY IJF UISTTE .03 QTII/HR/FTR/(DES F/FT) CAN WALL. IS .187 IN. THICK WITH THERMAL CUMDIJCTIVITY $=14.0$

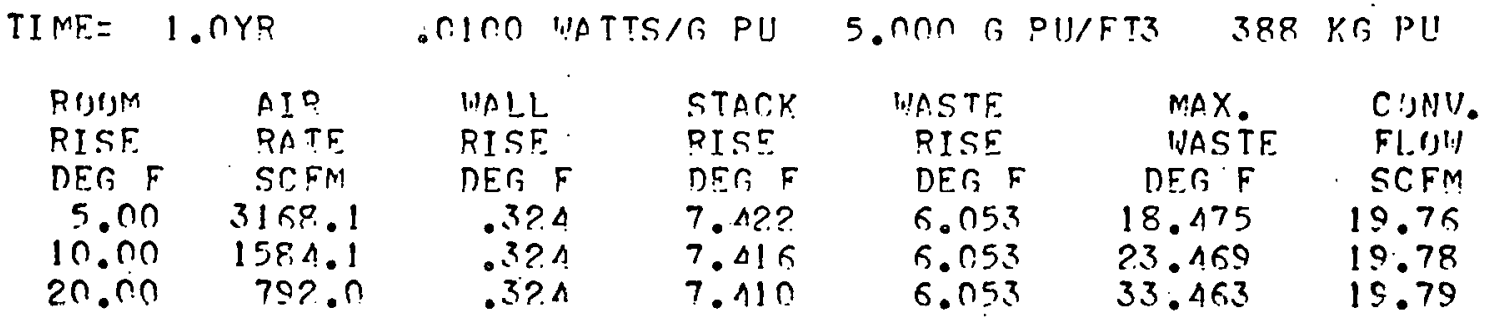

$\begin{array}{ll}\text { IIMF. }= & 10.0 Y P \\ \text { RIJIM } & \text { AIR } \\ \text { RISE } & \text { RATE } \\ \text { DEG F } & \text { SCFM } \\ 5.00 & 4153.7 \\ 10.00 & 2.076 .9 \\ 20.00 & 1038.4\end{array}$

TIME $=50.0 Y R$

$\begin{array}{ll}\text { ROSNM } & \text { AIR } \\ \text { RISF } & \text { RATE } \\ \text { DES F } & \text { SCFM } \\ 5.00 & 5507.3 \\ 10.00 & 2.753 .6 \\ 20.00 & 1376.8\end{array}$

TIME $=100 . O Y R$

$\begin{array}{llll}\text { RIJJM } & \text { AIR } & \text { WALL } & \text { STACK } \\ \text { RISE } & \text { RATF } & \text { RISF } & \text { RISE } \\ \text { DEGF } & \text { SCFM } & \text { DESF } & \text { DEGF } \\ 5.00 & 5153.0 & .479 & 10.695 \\ 10.00 & 2576.5 & .479 & 10.686 \\ 20.00 & 1288 . ? & .478 & 10.677\end{array}$
NIJW AT END

. I132. WATTS/G PIJ

$\begin{array}{ll}\text { WALL } & \text { STACK } \\ \text { PISE } & \text { RISE } \\ \text { DEGF } & \text { DESF } \\ .403 & 9.095 \\ .403 & 9.089 \\ .402 & 9.081\end{array}$

.0175 WATTS/G PU

$\begin{array}{ll}\text { WALL } & \text { STACK } \\ \text { RISE } & \text { RISE } \\ \text { DEGF } & \text { DEG F } \\ .505 & 11.2 .43 \\ .505 & 11.233 \\ .504 & 11.22 .4\end{array}$

.0163 WATTS/G PU

16:2.3 RAN O MINS 0.28 S.TCS

$$
\begin{aligned}
& 5.000 \text { G PU/FT3 } 388 \text { KEPU } \\
& \begin{array}{ccc}
\text { WASTE } & \text { MAX. } & \text { CIJNY. } \\
\text { RISE } & \text { WASTE } & \text { FLIJU } \\
\text { DEG.F } & \text { DEGF } & \text { SCFM } \\
7.936 & 22.033 & 2.1 .14 \\
7.936 & 27.02 .5 & 21.16 \\
7.936 & 37.017 & 21.18
\end{array} \\
& 5.000 \text { G PU/FT3 388 KG PU } \\
& \begin{array}{ccc}
\text { WASTE } & \text { MAX. } & \text { CUNY. } \\
\text { RISE } & \text { WASTE } & \text { FLYWW } \\
\text { DEGF } & \text { DEG F } & \text { SCFM } \\
10.52 .2 & 26.765 & 2.2 .68 \\
10.522 & 31.755 & 22.70 \\
10.522 . & 41.746 & 22.72
\end{array}
\end{aligned}
$$

5. ก0n G PU/FT3 38, KG PU

$\begin{array}{lcr}\text { WASTE } & \text { MAX. } & \text { CUNY. } \\ \text { RISE } & \text { WASTE } & \text { FLUW } \\ \text { DESF } & \text { DEGF } & \text { SCFM } \\ 9.845 & 2.5 .540 & 2.2 .31 \\ 9.845 & 30.531 & 22.33 \\ 9.845 & 10.522 & 2.2 .34\end{array}$




\title{
TABLE B.2. (Continued)
}

ALPHAI

\author{
$16: 01 \quad 05 / 2.7 / 72$ \\ ALPHA WASTE STIJRAGE FACILITY \\ HEAT TRANSFER ANALYSIS
}

TIJTAL NUMRER UF RARRFISS= 54 IN COJIFIFLIPATIUN 9 BY 6 BY $10 \mathrm{HIGH}$ BAPRELS ARE 6 FT BY 4 FT BY 6 FT LUNF UITH .?5 FT RADIUS AT CIJRERS SPACING IS 10 FT HIJRIZUNTAL ANI 0.33 FT VERTISAL

SURFACE AREA UF PIJIM $=15000$ FT? WITH I FT THICK WALLS WITH $K=.00$.

VIJUUME UF BARREL 143.7 FT3, TITAL WASTE VILUUME $=77586$ FT3

THEPMAL CONIIUCTIUITY UF WASTE .03 RTU/HR/FT2/(DEG F/FT)

CAN WALL IS .187 IN. THICK WITH THERMAL CHNDIJCTIUITY $=14.0$

\begin{tabular}{|c|c|c|c|c|c|c|c|}
\hline \multicolumn{2}{|c|}{ TIME: $1 . \cap Y R$} & \multicolumn{2}{|c|}{. OIOO WATTS/G PU } & \multicolumn{2}{|c|}{5.000 Fi $P U / F T 3$} & 388 & $K G P U$ \\
\hline $\begin{array}{l}\text { RIJIJM } \\
\text { RISE } \\
\text { DEG F } \\
5.00 \\
10.00 \\
20.00\end{array}$ & $\begin{array}{l}\text { AIR } \\
\text { RATE } \\
\text { SCFM } \\
3168.1\end{array}$ & 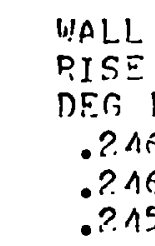 & $\begin{array}{l}\text { STACK } \\
\text { RISE } \\
\text { DEG F } \\
10.868 \\
10.852 \\
10.819\end{array}$ & $\begin{array}{l}\text { WASTE } \\
\text { RISE } \\
\text { DFE } \\
6.053 \\
6.053 \\
6.053\end{array}$ & & $\begin{array}{l}x . \\
\text { SiF } \\
\text { SF } \\
01 \\
11 \\
\text { n? }\end{array}$ & $\begin{array}{l}\text { CINV. } \\
\text { FLUW } \\
\text { SCFM } \\
26.99 \\
27.02 \\
2 \% .04\end{array}$ \\
\hline \multicolumn{2}{|c|}{ TIME: $=10.0 Y R$} & \multicolumn{2}{|c|}{.0132 WATTS/G PU } & \multicolumn{2}{|c|}{5.000 S $P U / F T 3$} & 388 & $K G P U$ \\
\hline $\begin{array}{l}\text { RUIJ!! } \\
\text { RISE } \\
\text { DES F F } \\
5.00 \\
10.00 \\
20.00\end{array}$ & $\begin{array}{c}\text { AIR } \\
\text { RATF } \\
\text { SCFP } \\
4153.7\end{array}$ & $\begin{array}{l}\text { WALL } \\
\text { RISE } \\
\text { DES } \\
.305 \\
.305 \\
.305\end{array}$ & $\begin{array}{l}\text { STACK } \\
\text { RISE } \\
\text { DEG F } \\
13.319 \\
13.308 \\
13.297\end{array}$ & $\begin{array}{l}\text { WASTF } \\
\text { RISF } \\
\text { DE. } \\
7.936 \\
7.936 \\
7.936\end{array}$ & & 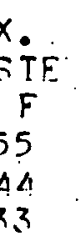 & $\begin{array}{l}\text { CUNV. } \\
\text { FLUW } \\
\text { SCFM } \\
28.88 \\
28.90 \\
28.92\end{array}$ \\
\hline \multicolumn{2}{|c|}{ TIME $=50.0 \mathrm{YR}$} & \multicolumn{2}{|c|}{. 175 WATTS/G PU } & \multirow{2}{*}{$\begin{array}{c}5.000 \mathrm{G} \\
\text { WASTS } \\
\text { RISE } \\
\text { DES } \\
10.522 \\
10.52 ? \\
10.522\end{array}$} & $P U / F T 3$ & 388 & $K G P U$ \\
\hline $\begin{array}{l}\text { RIJUM } \\
\text { RISE } \\
\text { DEG F } \\
5.00 \\
10.00 \\
20.00\end{array}$ & $\begin{array}{c}\text { AIR } \\
\text { RATE } \\
\text { SCFM } \\
5507.3 \\
2.753 .6 \\
1376.8\end{array}$ & $\begin{array}{l}\text { WALL } \\
\text { RISE } \\
\text { DEG } \\
.383 \\
.388 \\
.388\end{array}$ & $\begin{array}{l}\text { STACK } \\
\text { RISE } \\
\text { DEG F } \\
16 . \Delta 62 \\
16.448 \\
16.434\end{array}$ & & & $\begin{array}{l}\text { SiTE } \\
54 \\
6 \\
6\end{array}$ & $\begin{array}{l}\text { Cony. } \\
\text { FLOW } \\
\text { SCFM } \\
30.08 \\
31.00 \\
31.03\end{array}$ \\
\hline \multicolumn{2}{|c|}{ TIME $=100.0 Y R$} & \multicolumn{2}{|c|}{.0163 WATTS/G PU } & $5.000 \quad G$ & $\mathrm{PU} / \mathrm{FT} 3$ & 388 & $K G P U$ \\
\hline $\begin{array}{l}\text { RIJUM } \\
\text { RISE } \\
\text { DES F F } \\
5.00 \\
10.00 \\
20.00\end{array}$ & $\begin{array}{c}\text { AIR } \\
\text { RATE } \\
\text { SCFM } \\
5153.0 \\
2.576 .5 \\
1288.2\end{array}$ & $\begin{array}{l}\text { WALL } \\
\text { RISE } \\
\text { DEF } \\
.363 \\
.363 \\
.363\end{array}$ & $\begin{array}{l}\text { STACK } \\
\text { RISE } \\
\text { DEG F } \\
15.5 S O \\
15.546 \\
15.633\end{array}$ & $\begin{array}{l}\text { WASTE } \\
\text { RISE } \\
\text { DEG F } \\
9.8 \Delta 5 \\
9.845 \\
9.845\end{array}$ & & $\begin{array}{l}X . \\
\text { STE } \\
5 \\
05 \\
92 \\
78\end{array}$ & $\begin{array}{l}\text { CIJNY. } \\
\text { FLUW } \\
\text { SCFM } \\
30.47 \\
30.49 \\
30.5 ?\end{array}$ \\
\hline
\end{tabular}

NIS'N AT E.ND

16:05 RAN O MINS 0.48 SECS 
TABLE B.3. TEMPERATURES IN A FACILITY WITH HEAT LOSSES THROUGH A I-FT THICK CONCRETE WALL

ALPHAI

$15: 55 \quad 0.5 / 2.7 / 7 ?$

ALFHA WASTE. STURAFF. FACILITY

HEAT TRAMISFER ANALYSIS

TUTAL NUMBER IJF BARRFLS= 540 IN CUNFI GUPATIUH S RY 6 RY IO HIGH BARRELS ARE ST FT RY A FT RY 6 FT LIJNG WITH .2.5 FT RAIIUS AT CIJRNERS SPACING IS .OO FT HURITINTAL AND 0.33 FT VERTICAL

SLIRFACE APFA IF RUIJM= 15000 FT? WITH 1 FT THICK WALLS WITH K= .50

VIJLIJME UF BAPREL $=143.7$ FT3, TUTAL WASTF. VULUME= 7758.6. FT3

THERMAL CUVDUCTIVITY UF WASTE .O3 RTU/HR/FT2/(DEG F/FT)

CAN WALL IS .187 IN. THICK WITH THERMAL CUVOUCTIVITY $=14.0$

TIME: $=1 . \cap Y Y^{\prime}$

.0100 HATTS/R PU

5. 000 i PII/FT3

388 KG PU

$\begin{array}{lllllll}\text { RIJIM } & \text { AIR } & \text { INALL } & \text { STACK } & \text { HASTE } & \text { MAX. } & \text { CUNW. } \\ \text { RISE } & \text { RATF } & \text { RISF } & \text { RISE } & \text { RISE } & \text { WASTE } & \text { FLUH } \\ \text { DEG F } & \text { SCFM } & \text { DESF } & \text { DEGF } & \text { DEG } & \text { DEG F } & \text { SCFM } \\ 1.77 & .0 & .096 & 98.087 & 11.046 & 110.906 & 2.99 \\ 1.77 & .0 & .096 & 98.087 & 11.046 & 110.906 & 2.99 \\ 1.77 & .0 & .096 & 98.087 & 11.046 & 110.906 & 2.99\end{array}$

IIME $=10.0 \% ?$

.0132 . WATS/G PU

$\begin{array}{ll}\text { RIJUM } & \text { AIR } \\ \text { RISE } & \text { RATE } \\ \text { DEG F } & \text { SCFII } \\ 2.33 & -0 \\ 2.33 & \text {. } \\ 2.33 & \text {. }\end{array}$

$\begin{array}{ll}\text { WALL } & \text { STACK } \\ \text { RISE } & \text { RISE } \\ \text { DES F DFF } \\ \text { 119 } \\ 1190.214 \\ 119 & 120.214 \\ 119 & 120.214\end{array}$

5.000

G $P[1 / F T 3$

$388 \quad \mathrm{KG} P \mathrm{PU}$

TIME $=50.0 Y R$

.0175 WATTS/G PU

$\begin{array}{ll}\text { RUIMM } & \text { AIR } \\ \text { RISE } & \text { RATE } \\ \text { DEG F } & \text { SCFM } \\ 3.08 & . n \\ 3.08 & \text { ? } \\ 3.08 & : 0\end{array}$

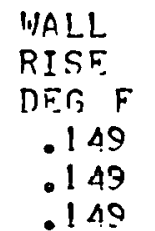

WASTE

RISE DEG. F

14. 482 .

14.482

14.482 .

MAX.
WASTE
DEG F
137.022 .2
137.022
137.022

C (J) N).

FLUU

SCFM

3.20

3.20

3.20

5. OnN G PU/FT3 388 KG PU

TI ME $=100.0 Y R$

$$
.0163 \text { WATTS/G PIJ }
$$

$\begin{array}{lr}\text { RISUM } & \text { AIP } \\ \text { RISE } & \text { RATE } \\ \text { DEG F } & \text { SCFM } \\ 2.89 & .0 \\ 2.89 & .0 \\ 2.89 & .0\end{array}$

$\begin{array}{lc}\text { HALL } & \text { STACK } \\ \text { RISE } & \text { RISE } \\ \text { DEG.F } & \text { DFG F } \\ .142 & 141.338 \\ .142 . & 141.338 \\ .142 & 141.338\end{array}$

148.575

140.575

148.575

WASTE

RISE

DEF F

19.201

13.201

19.201

$\begin{array}{rl}\text { MAX. } & \text { CUNV. } \\ \text { UIASTE } & \text { FLIJW } \\ \text { DEG F } & \text { SCFM } \\ 170.861 & 3.43 \\ 170.861 & 3.43 \\ 170.861 & 3.43\end{array}$

NIJ) AT END

$$
5.000
$$

G PU/FT3

$388 K G \quad P U$

$16: 00$

$$
\text { RAN O MINS }
$$

0.48 SECS

$\begin{array}{rl}\text { MAX. } & \text { CUNV. } \\ \text { WASTF } & \text { FLUW } \\ \text { DEG F } & \text { SC.FM } \\ 162.139 & 3.38 \\ 162.189 & 3.38 \\ 162.189 & 3.38\end{array}$




\section{TABLE B.3. (Continued)}

RU1+CLE 710,790

READY

RUN

ALPHAI

$$
16: 51 \quad 0.5 / 2.7 / 72
$$

\section{ALPHA WASTE STIJRAGE FACILITY}

HEAT TRANSFER A NALYSIS

TUTAL NIJMBER UF BARRELS= 540 IN CUNFIGIJPATIUN 9 BY 6 RY 10 HIGH BARRELS ARE 6 FT BY 4 FT PYY 6 FT LUNI WITH .25 FT RADIUS AT CURNERS SPACING IS .OO FT HURIZUNTAL AMD 0.33 FT VERTICAL

SLIPFACE. AREA UF RIJM: 15000 FT? WITH 2. FT THICK WALLS WITH K= .25

VILLUME UF RARRELL 143.7 FT3, TUTAL WASTE VISUUME: 77586 FT3

THERIALL CONDIJCTIVITY UF UIASTE .03 RTIJ/HR/FT2/(DEG. F/FT)

CAN WALL IS .187 IN. THICK WITH THERMAL CUNDUCTIVITY $=14.0$

TIME $=1.0 Y R$

$\begin{array}{ll}\text { RUUM } & \text { AIR } \\ \text { RISE } & \text { RATF } \\ \text { DEG.F } & \text { SCFM } \\ 5.00 & 936.0 \\ 7.10 & .0 \\ 7.10 & .0\end{array}$

$$
.0100 \text { hatTS/g PU }
$$

$$
5.000
$$

f. $P U / F T 3$

388. KG PU

TIME $=10.0 Y R$

$\begin{array}{ll}\text { WALL } & \text { STACK. } \\ \text { RISE } & \text { R.ISE } \\ \text { DEG F } & \text { DEG F } \\ .096 & 98.584 \\ .097 & 98.753 \\ .097 & 98.753\end{array}$

WASTE
RISE
DES F
11.046
11.046
11.046

MAX.
UASTE
DEG F
114.630
116.895
116.895

Cisñ.

FLIII

SCFM

2.98

11.046

2.97

.0132 HATTS/G PU

5.000

G PU/FT3

388 KG PU

$\begin{array}{lr}\text { RUISIM } & \text { AIR } \\ \text { RISE } & \text { RATE } \\ \text { DEG F } & \text { SCFM } \\ 5.00 & 192.1 .5 \\ 9.30 & .0 \\ 9.30 & .0\end{array}$

$\begin{array}{lr}\text { WALL } & \text { STACK } \\ \text { RISE } & \text { RISE } \\ \text { DESF } & \text { DEGF } \\ .120 & 120.564 \\ .120 & 12.1 .030 \\ .120 & 121.030\end{array}$

WASTE
RISE
DESTF
14.482
14.482.
14.482

MAX.
WASTE
DEG
140.146
144.816
144.816

CISNV. FL.J', SCFM

3.19

3.18

TIME $=50.0 Y R$

.0175 WATTS/R PU

5.000 G PU/FT3

$388, K G P U$

\begin{tabular}{lr} 
RIJUM & \multicolumn{1}{c}{ AIP } \\
RISE. & RATE \\
DEG F & SCFM \\
5.00 & 32.75 .2 \\
10.00 & 52.1 .5 \\
12.34 & .0
\end{tabular}

$\begin{array}{lr}\text { WALL } & \text { STACK } \\ \text { RISE } & \text { RISE } \\ \text { DEG F } & \text { DEF F } \\ .150 & 148.926 \\ .147 & 145.636 \\ .147 & 145.598\end{array}$

WASTE
RISE
DEF F
19.201
19.201
19.201

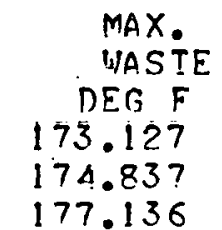

CIJNY.
FLOW
SCFM
3.42
$3.5 U$
$3.5 n$

TIME $=100.0 Y R$

$$
.0163 \text { WATTS/G PU }
$$

5.000 G PU/FT3

388 KG PU

$\begin{array}{lr}\text { RIJUM } & \text { AIR } \\ \text { RISE } & \text { RATF } \\ \text { DEG F } & \text { SCFM } \\ 5.00 & 292.0 .8 \\ 10.00 & 344.3 \\ 111.54 & .0 \\ \text { IIT } & .\end{array}$

$\begin{array}{lc}\text { WALL } & \text { STACK } \\ \text { RISE } & \text { RISE } \\ \text { DEG F } & \text { DEFF } \\ .142 & 141.717 \\ .139 & 138.541 \\ .139 & 138.517\end{array}$

WASTE
RISE
DEG F
17.966
17.966
17.966

MAX.
WASTE
DEG F
164.683
166.507
168.025

CISNY.
FLOW
SCFM
3.37
3.44
3.44

16:59 RAN O MINS 0.48 SECS 
APPENDIX C

LISTING OF CONVERSATIONAL BASIC LANGUAGE COMPUTER PROGRAM

ALPHA 1

FOR USE IN .HEAT TRANSFER CALCULATIONS IN DESIGN

OF AN ALPHA WASTE STORAGE FACILITY 


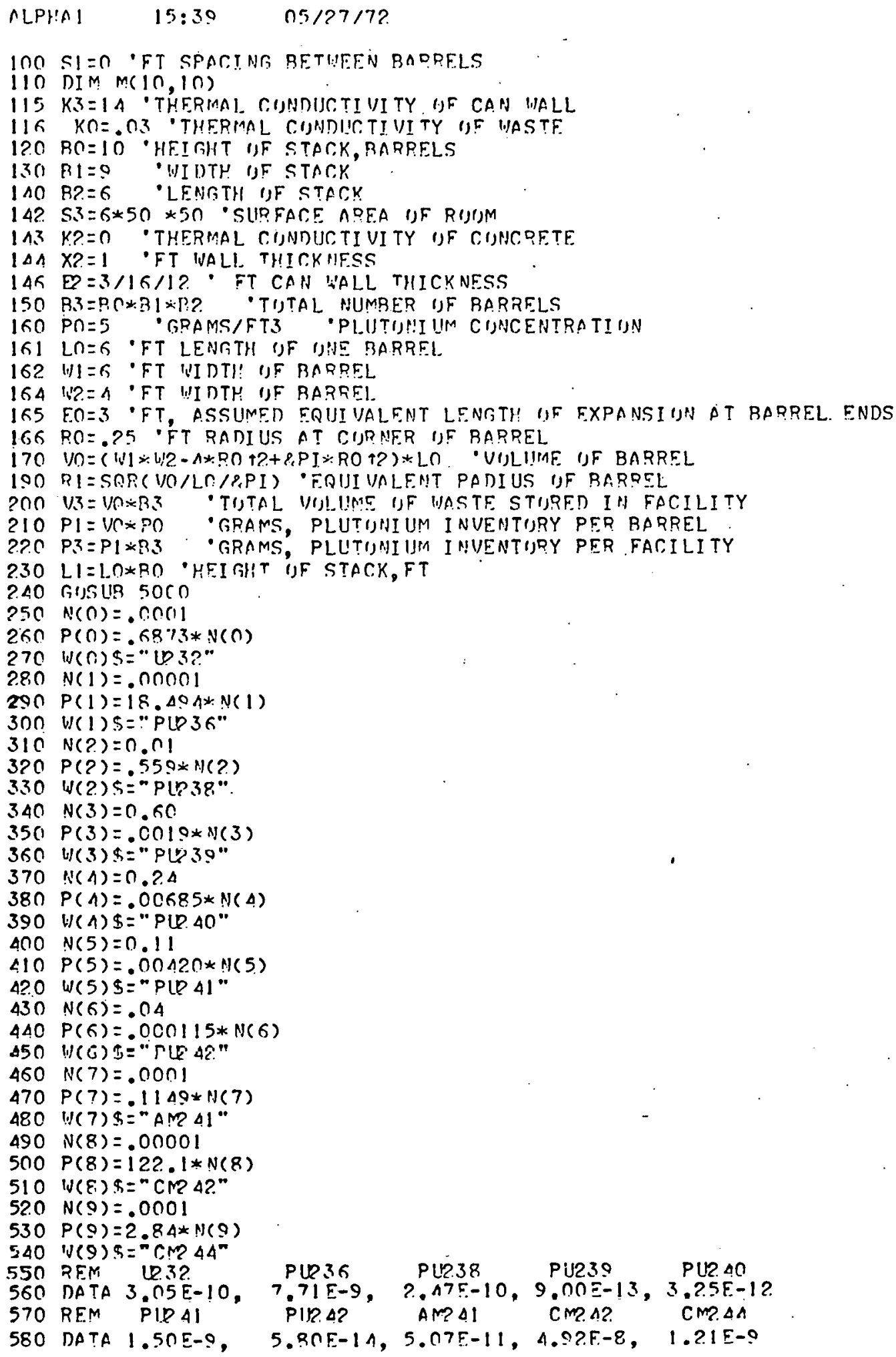




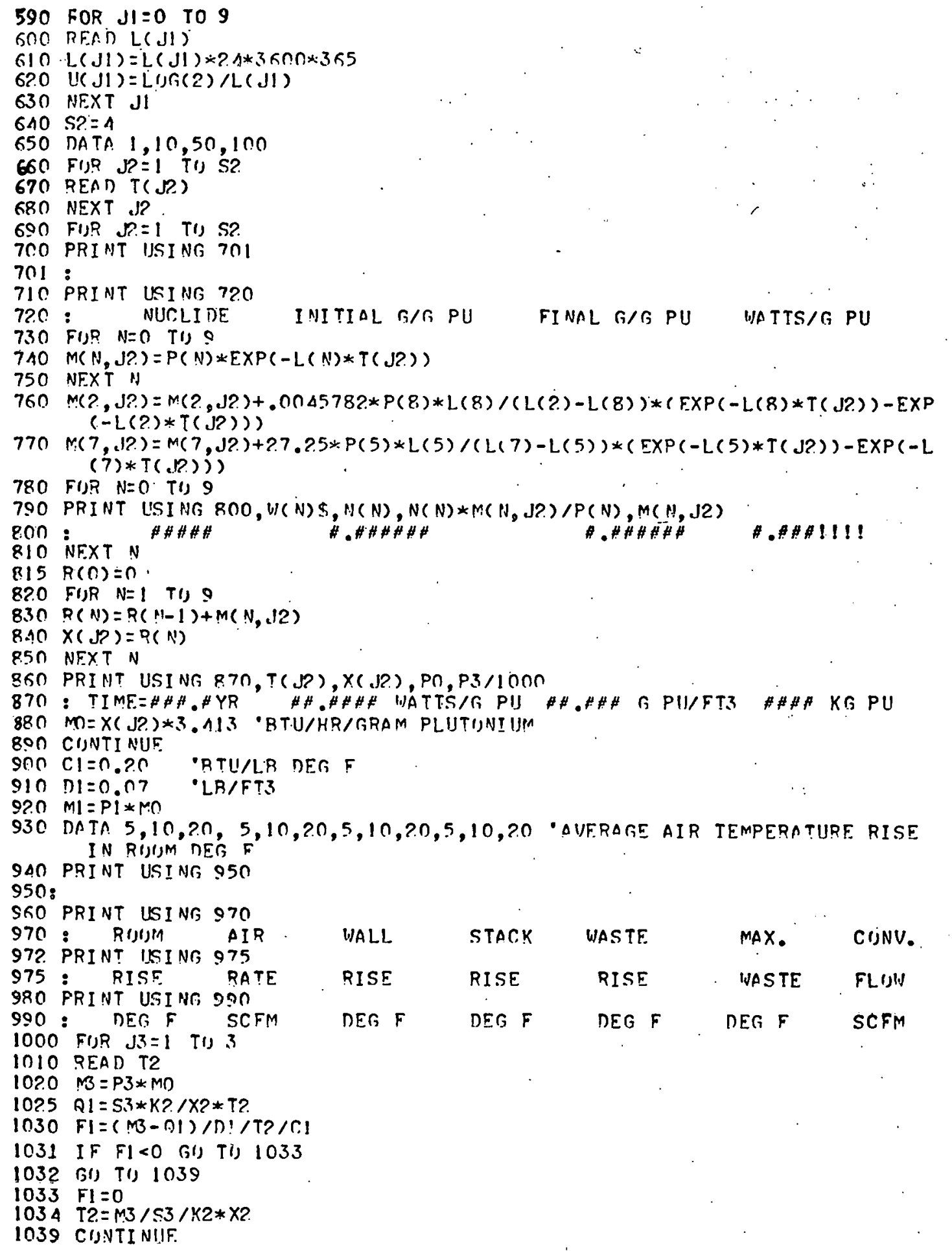

$\begin{array}{lllll}\text { WALL } & \text { STACK } & \text { WASTE } & \text { MAX. } & \text { CIJNV. } \\ \text { RISE } & \text { RISE } & \text { RISE } & \text { WASTE } & \text { FLIJN } \\ \text { DES F } & \text { DEGF } & \text { DEG F } & \text { DEG F } & \text { SCFM }\end{array}$




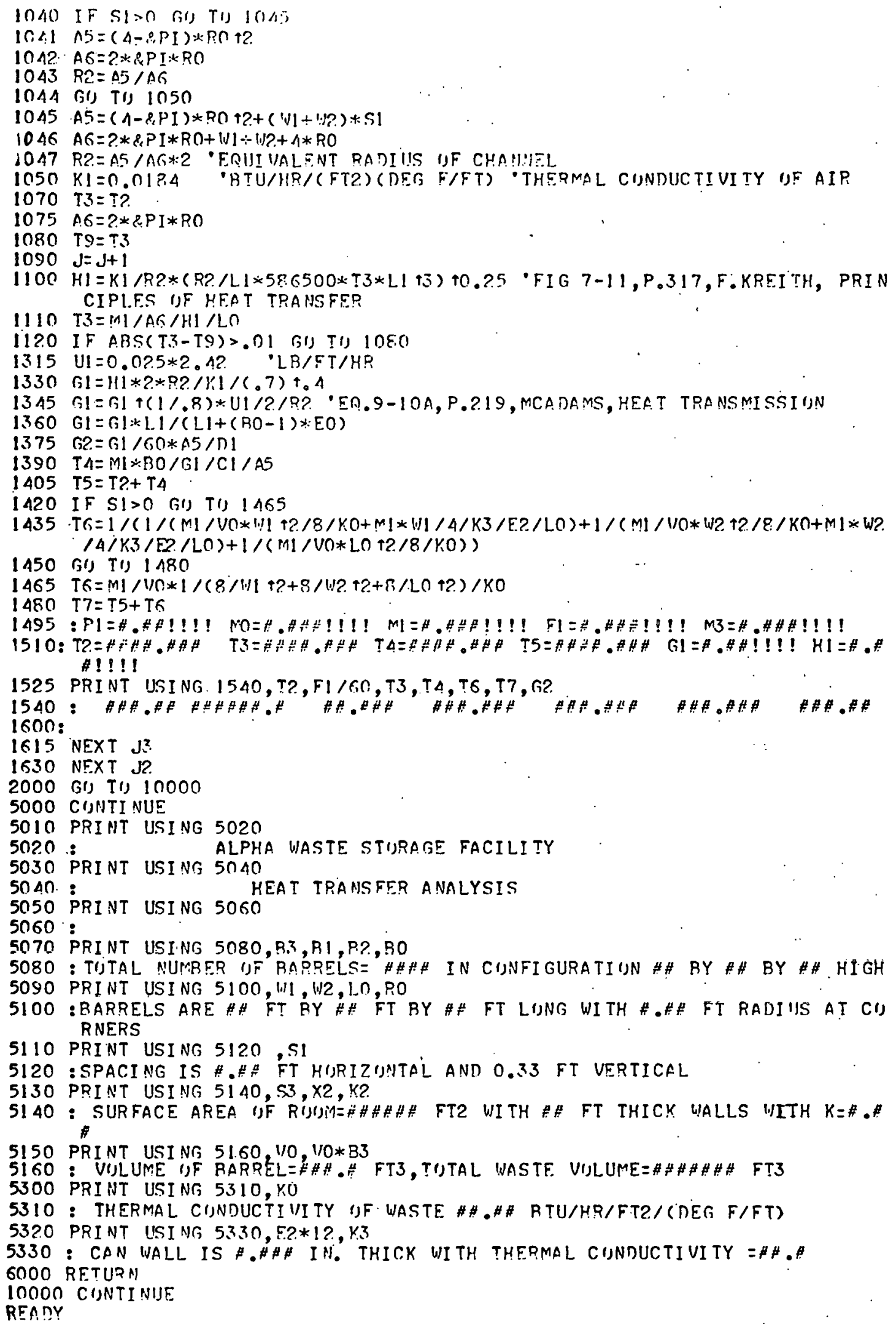


No. of

Copies

OFFSITE

1 AEC Chicago Patent Group

U.S. Atomic Energy Commission

Argonne, IL 60439

A. A. Churm .

1 AEC Headquarters

Division of Operational Safety

Germantown, Maryland

M. W. Tiernan

2 AEC Technical Information Center

ONSITE

2 AEC Richland Operations Office

R. B. Goranson

B. J. Melton $/$ P. F. X. Dunigan, Jr.

3 Atlantic Richfield Hanford Co.

D. E. Larson

D. C. Nelson

D. D. Wodrich

24 Battelle-Northwest

H. C. Burkholder

N. E. Carter

G. J. Dau

J. W. Finnigan

G. Jansen

P. J. Pelto

A. M. Platt

K. J. Schneider

J. R. Sheff

T. H. Smith

Technical Information Files

Technical Publications

Distr.1 